\title{
The onset of electrothermoconvection in a rotating Brinkman porous layer
}

\author{
I. S. Shivakumara ${ }^{\mathrm{a}}$, Chiu-On $\mathrm{Ng}^{*}$ and M.S. Nagashree ${ }^{\mathrm{b}}$ \\ *Department of Mechanical Engineering, The University of Hong Kong, Pokfulam Road, \\ Hong Kong. Email: cong@hku.hk \\ a Permanent address: UGC-CAS in Fluid Mechanics, Department of Mathematics, \\ Bangalore University, Bangalore-560 001, India. Email: shivakumarais@gmail.com \\ ${ }^{\mathbf{b}}$ Department of Mathematics, Vijaya College, Bangalore-560 004, India
}

\begin{abstract}
The criterion for the onset of electrothermoconvection in a rotating horizontal layer of Brinkman porous medium is investigated for different types of velocity boundary conditions namely, (i) both boundaries stress-free, (ii) both boundaries rigid and (iii) lower rigid and upper free boundaries. Results indicate that the nature of boundaries and speed of rotation significantly influence the stability characteristics of the system. In the case of stress-free condition, it is shown that the necessary conditions for the occurrence of oscillatory onset are independent of an external electric field. Contrary to their stabilizing effect in the absence of rotation, increasing the ratio of viscosities $\Lambda$ and decreasing the Darcy number $D a$ show some destabilizing effect on the onset of stationary electrothermoconvection in the presence of rotation and some important observations are made on the stability characteristics of the system. Moreover, the similarities and differences between free-free, rigid-rigid and rigidfree boundaries are emphasized in triggering the onset of electrothermoconvection in a rotating porous layer. For small Taylor number domain, the stress-free boundaries is found to be always unstable than that of rigid-rigid and rigid-free boundaries. However, this trend is reversed at higher Taylor number domain because the stability of the stress-free case is increased more quickly than the other boundaries.
\end{abstract}




\section{Introduction}

The study of thermogravitational convection in a rotating fluid layer heated from below has attracted researchers since it is a relevant topic for meteorologists, climatologists, oceanographers and astrophysicists alike. The problem is also of importance in many engineering applications and copious literature is available on thermal convection in a rotating fluid layer (Chandrasekhar [1], Galdi and Straughan [2], Kloosterziel and Carnevale [3] and references therein). The corresponding problem in a rotating layer of porous medium has also received considerable attention in the literature. Patil and Vaidyanathan [4] have studied thermal convection in a rotating fluid saturated porous layer under the influence of variable viscosity using the Brinkman model. Palm and Tyvand [5] have used the Darcy model to study the linear stability problem of thermal convection in a rotating porous layer and they have shown that their results are equivalent to those of non-rotating anisotropic porous medium case. Using the Brinkman model, Qin and Kaloni [6] have studied the nonlinear stability of a rotating porous layer by including the convective inertia term in the Brinkman model. Vadasz [7] has used both linear and weak nonlinear theories to study the effect of Coriolis force on gravity-driven convection in a rotating porous layer heated from below by employing the modified Darcy model. An excellent review of research on thermal convection in a rotating porous medium is given by Vadasz [8]. A nonlinear stability analysis for thermal convection in a rotating porous layer has been performed by Straughan [9]. The problem of onset of thermal convection in a rotating porous medium bounded between rigid boundaries has been considered by Desaive et al.[10]. Shivakumara et al. [11] have investigated linear and weakly nonlinear thermal convection in a rotating porous layer and they have shown that decrease in the permeability and increase in the effective viscosity of the fluid have a destabilizing effect on the onset of stationary convection at high rotation rates. Recently, Falsaperla et al. [12] have considered the problem of thermal convection in a rotating horizontal layer of porous medium with Newton-Robin type of temperature boundary conditions.

It is recognizable that many convective instability problems of practical importance involve electrically conducting fluids. In such cases, the effect of external fields like magnetic and electric fields become important. In particular, the magnetic field effects become dominant if the fluid is highly electrically conducting. To the contrary, if the fluid is dielectric with low electrical conductivity then the electric forces play a major role in driving 
the motion. Several studies have been carried out to assess the effect of AC and DC electric fields on natural convection due to the fact that many problems of practical importance involve dielectric fluids. In these fluids, an applied temperature gradient produces nonuniformities in the electrical conductivity and/or the dielectric permittivity. The variation of electrical conductivity of the fluid with temperature produces free charges in the bulk of the fluid. These free charges interacting with applied or induced electric field produce a force that eventually causes fluid motion. On the other hand, when there is variation in dielectric permittivity and the electric field is intense then the polarization force which is induced by the non-uniformity of the dielectric constant causes fluid motion. In either case, convection can occur in a dielectric fluid layer even if the temperature gradient is stabilizing and such an instability produced by an electric field is called electroconvection, which is analogous to Rayleigh-Benard convection. In addition, if the applied temperature gradient is also destabilizing then such an instability problem is called electrothermoconvection.

Natural convection problem under an AC and/or DC electric field has been studied extensively and an exhaustive review on this topic has been given by Jones [13] and Saville [14]. The combined effects of DC electric field and volumetric heat source on the onset of convection in a dielectric fluid layer heated from below is investigated by Shivakumara et al. [15], while the influences of vertical AC electric field as well as internal heat generation on the onset of electrothermoconvection in a horizontal dielectric fluid layer is analyzed by Shivakumara et al. [16]. A more detailed analysis on EHD instability in a horizontal fluid layer with electrical conductivity gradient subject to a weak shear flow is presented by Chang et al. [17]. Studies have also been undertaken in the past to understand the effect of rotation on electroconvection. Takashima [18] was the first to consider the effect of rotation on the onset of instability in a dielectric fluid layer under the action of a vertical AC electric field and a vertical temperature gradient. The influences of an AC electric field and rotation on Benard-Marangoni instability in a layer of an incompressible fluid with small electrical conductivity are investigated by Douiebe et al. [19]. Othman [20] has studied the stability of a rotating layer of viscoelastic dielectric liquid heated from below. Recently, Ruo et al. [21] have considered the EHD instability of a horizontal rotating fluid layer with a vertical electrical conductivity gradient for different kinds of velocity boundary conditions.

Electroconvection in a dielectric fluid saturated porous medium is of particular interest in a geophysical system, since the electric field can provide the driving force in 
laboratory models of thermal convection of electrically conducting fluids in the earth's core. Besides, the study of electric field on a fluid flow in porous media is of particular importance in view of its possibility of reduction of fluid viscosity in enhancing petroleum production (Moreno et al., [22]). Recently, electroconvection in a horizontal dielectric fluid saturated densely packed porous layer under the simultaneous action of vertical electric field and vertical temperature gradient when the walls of the layer are subjected to time periodic temperature modulation is investigated by Rudraiah and Gayathri [23].

Under the circumstances, the rotational effect seems to be of significance on ETC in porous media and has not been given any attention in the literature. Therefore, the aim of the present paper is to bring forth the salient features of the combined effects of rotation and $\mathrm{AC}$ electric field on the onset of convection in a dielectric fluid saturated Brinkman porous medium. To encompass both geophysical and laboratory problems, the stability analysis is carried out for three different types of velocity boundary conditions namely, (i) both boundaries free, (ii) both boundaries rigid and (iii) lower boundary is rigid and upper boundary is free. Equations for more general forms of convection in porous media are derived by Subramanian and Rajagopal [24], Kannan and Rajagopal [25], Rajagopal et al. [26] and it will be interesting to analyze the problem in these theories. However, in the present study a modified Lapwood-Brinkman extended Darcy model with fluid viscosity different from effective viscosity is used to describe the flow in the porous medium. An exact solution to the resulting eigenvalue problem is obtained in the case of stress-free boundaries, while for the other two boundary combinations the critical stability parameters are obtained numerically using the Galerkin-type of weighted residuals method. The effects of various physical parameters on the stability of the system are analyzed.

\section{Mathematical formulation}

The physical configuration is as shown in Fig.1. It consists of a dielectric fluid saturated sparsely packed horizontal porous layer of thickness $d$ with a uniform vertical AC electric field applied across the porous layer which is kept rotating about the vertical axis with constant angular velocity $\vec{\Omega}=(0,0, \Omega)$. The lower and upper boundaries of the porous layer are maintained at uniform, but different temperatures $T_{0}$ and $T_{1}\left(<T_{0}\right)$ respectively, and thus a constant temperature difference $\Delta T\left(=T_{0}-T_{1}\right)$ is maintained between the boundaries. A Cartesian coordinate system $(x, y, z)$ is chosen with the origin at the bottom of the porous 
layer and the z- axis normal to the porous layer in the gravitational field. Besides, it is assumed that the fluid and solid matrix are in local thermal equilibrium and rotation does not disrupt the isotropy of the porous medium.

The relevant basic equations under the Oberbeck-Boussinesq approximation are:

$$
\begin{aligned}
& \nabla \cdot \vec{q}=0 \\
& \rho_{0}\left[\frac{1}{\phi} \frac{\partial \vec{q}}{\partial t}+\frac{1}{\phi^{2}}(\vec{q} \cdot \nabla) \vec{q}+\frac{2}{\phi}(\vec{\Omega} \times \vec{q})\right]=-\nabla p+\rho \vec{g}-\frac{\mu}{k} \vec{q}+\tilde{\mu} \nabla^{2} \vec{q}+\vec{f}_{e} \\
& A \frac{\partial T}{\partial t}+(\vec{q} \cdot \nabla) T=\kappa \nabla^{2} T \\
& \rho=\rho_{0}\left\{1-\alpha\left(T-T_{0}\right)\right\}
\end{aligned}
$$

where $\vec{q}$ is the velocity vector, $T$ is the temperature, $p$ is the pressure, $\rho$ is the fluid density, $\kappa$ is the effective thermal diffusivity, $k$ is the permeability of the porous medium, $\mu$ is the fluid viscosity, $\tilde{\mu}$ is the effective viscosity, $\vec{g}$ is the acceleration due to gravity, $A$ is the ratio of heat capacities, $\phi$ is the porosity of the porous medium, $\alpha$ is the thermal expansion coefficient, $\rho_{0}$ is the density at reference temperature $T=T_{0}$ and $\vec{f}_{e}$ is the force of electrical origin which can be expressed as

$$
\vec{f}_{e}=\rho_{e} \vec{E}-\frac{1}{2} \vec{E} \cdot \vec{E} \nabla \varepsilon+\frac{1}{2} \nabla\left(\rho \frac{\partial \varepsilon}{\partial \rho} \vec{E} \cdot \vec{E}\right) .
$$

Here $\vec{E}$ is the electric field, $\rho_{e}$ is the charge density and $\varepsilon$ is the dielectric constant. In Eq. (5), the last electrostriction term can be grouped with the pressure $p$ in Eq. (2) and it has no effect on an incompressible fluid. The first term on the right hand side is the Coulomb force due to a free charge and the second term depends on the gradient of $\varepsilon$. The electrical force $\vec{f}_{e}$ will have no effect on the bulk of the dielectric fluid if both the dielectric constant $\varepsilon$ and the electrical conductivity $\sigma$ are homogeneous. Since $\varepsilon$ and $\sigma$ are functions of temperature, a temperature gradient applied to a dielectric fluid produces a gradient in $\varepsilon$ and $\sigma$. The application of a dc electric field then results in the accumulation of free charge in the liquid. The free charge increases exponentially in time with a time constant $\varepsilon / \sigma$, which is known as the electrical relaxation time. If an ac electric field is applied at a frequency much higher than the reciprocal of the electrical relaxation time, the free charge does not have time to accumulate. Moreover, the electrical relaxation times of most dielectric liquids appear to be sufficiently long to prevent the buildup of free charge at standard power line frequencies. At 
the same time, dielectric loss at these frequencies is so low that it makes no significant contribution to the temperature field [13]. Under the circumstances, only the force induced by non-uniformity of the dielectric constant is considered.

The relevant Maxwell equations are then

$$
\begin{aligned}
& \nabla \times \vec{E}=0 \\
& \nabla \cdot(\varepsilon \vec{E})=0 .
\end{aligned}
$$

In view of Eq.(6), $\vec{E}$ can be expressed as

$$
\vec{E}=-\nabla V
$$

where $V$ is the electric potential. The dielectric constant is assumed to be a linear function of temperature in the form

$$
\varepsilon=\varepsilon_{0}\left[1-\gamma\left(T-T_{0}\right)\right]
$$

where $\gamma(>0)$ is the thermal expansion coefficient of dielectric constant.

The basic state is quiescent and is given by

$$
\vec{q}=\vec{q}_{b}=0, T=T_{b}(z), p=p_{b}(z), \vec{E}=\vec{E}_{b}(z), \varepsilon=\varepsilon_{b}(z)
$$

where the subscript $b$ denotes the basic state. Substituting Eq.(10) in Eqs.(1) - (9), we get

$$
\begin{aligned}
& 0=-\nabla\left(\frac{p_{b}}{\rho_{0}}\right)+\frac{\rho_{b}}{\rho_{0}} \vec{g}+\nabla\left[\vec{E}_{b}\left(\frac{\partial \varepsilon_{b}}{\partial \rho}\right)\right]-\frac{\vec{E}_{b}^{2}}{2 \rho_{0}} \nabla \varepsilon_{b} \\
& \frac{d^{2} T_{b}}{d z^{2}}=0 \\
& \rho_{b}=\rho_{0}\left\{1-\alpha\left(T_{b}-T_{0}\right)\right\} \\
& \varepsilon_{b}=\varepsilon_{0}\left[1-\gamma\left(T_{b}-T_{0}\right)\right] \\
& \nabla \cdot\left(\varepsilon_{b} \vec{E}_{b}\right)=0 .
\end{aligned}
$$

Solving Eq.(11b) using the boundary conditions

$$
\begin{array}{ll}
T_{b}=T_{0} & \text { at } \quad z=0 \\
T_{b}=T_{1} & \text { at } \quad z=d
\end{array}
$$

we get

$$
T_{b}-T_{0}=-\Delta T z / d .
$$

In view of Eq.(11e) and noting that $E_{b x}=E_{b y}=0$, it follows that

$$
\varepsilon_{b} E_{b z}=\varepsilon_{0} E_{0}=\text { constant (say). }
$$

Then we have 


$$
E_{b z}=\frac{E_{0}}{1+\Delta T z / d}
$$

and hence

$$
V_{b}(z)=-\frac{E_{0} d}{\gamma \Delta T} \log (1+\gamma \Delta T z / d)
$$

where

$$
E_{0}=-\frac{V_{1} \gamma \Delta T / d}{\log (1+\gamma \Delta T)}
$$

is the externally applied electric field at $z=0$.

To study the stability of the basic state, we superimpose infinitesimally small perturbations $\left(\vec{q}^{\prime}, p^{\prime}, \vec{E}^{\prime}, T^{\prime}, \rho^{\prime}, \varepsilon^{\prime}\right)$ on the basic state in the form

$$
\vec{q}=\vec{q}^{\prime}, p=p_{b}+p^{\prime}, \vec{E}=\vec{E}_{b}+\vec{E}^{\prime}, T=T_{b}+T^{\prime}, \rho=\rho_{b}+\rho^{\prime}, \varepsilon=\varepsilon_{b}+\varepsilon^{\prime} .
$$

Substituting Eq.(18) into Eqs.(1) - (9), linearizing the equations by neglecting the products of primed quantities, eliminating the pressure from the momentum equation by operating curl twice and retaining the vertical component we get the required equations in the form

$$
\begin{aligned}
& \left(\frac{1}{\varphi} \frac{\partial}{\partial t}-\frac{\tilde{\mu}}{\rho_{0}} \nabla^{2}+\frac{\mu}{\rho_{0} k}\right) \nabla^{2} w^{\prime}=\alpha g \nabla_{h}^{2} T^{\prime}-\frac{2 \Omega}{\varphi} \frac{\partial \xi^{\prime}}{\partial z}+\nabla_{h}^{2}\left[\frac{E_{0} \varepsilon_{0} \gamma}{\rho_{0}}\left(\frac{-\Delta T}{d}\right)\left(\gamma E_{0} T^{\prime}-\frac{\partial V^{\prime}}{\partial z}\right)\right] \\
& \left(\frac{1}{\phi} \frac{\partial}{\partial t}-\frac{\tilde{\mu}}{\rho_{0}} \nabla^{2}+\frac{\mu}{\rho_{0} k}\right) \xi^{\prime}=\frac{2 \Omega}{\phi} \frac{\partial w^{\prime}}{\partial z} \\
& \left(A \frac{\partial}{\partial t}-\kappa \nabla^{2}\right) T^{\prime}=\frac{\Delta T}{d} w^{\prime} \\
& \nabla^{2} V^{\prime}=\eta E_{0} \frac{\partial T^{\prime}}{\partial z}
\end{aligned}
$$

where $\xi^{\prime}=\partial v^{\prime} / \partial x-\partial u^{\prime} / \partial y$ is the vertical component of perturbed vorticity vector. Nondimensionalizing Eqs.(19)- (22) by scaling $(x, y, z)$ by $d$, t by $d^{2} / \kappa, \vec{q}^{\prime}$ by $\kappa / d, \xi^{\prime}$ by $\kappa / d^{2}, T^{\prime}$ by $\Delta T$ and $V^{\prime}$ by $\gamma E_{0} \Delta T d$, we obtain ( after neglecting the primes for simplicity)

$$
\begin{aligned}
& \left(\frac{1}{\operatorname{Pr}} \frac{\partial}{\partial t}-\Lambda \nabla^{2}+D a^{-1}\right) \nabla^{2} w=R_{t} \nabla_{h}^{2} T-T a^{1 / 2} \frac{\partial \xi}{\partial z}+R_{e a} \nabla_{h}^{2}\left(T-\frac{\partial V}{\partial z}\right) \\
& \left(\frac{1}{\operatorname{Pr}} \frac{\partial}{\partial t}-\Lambda \nabla^{2}+D a^{-1}\right) \xi=T a^{1 / 2} \frac{\partial w}{\partial z} \\
& \left(A \frac{\partial}{\partial t}-\nabla^{2}\right) T=w
\end{aligned}
$$




$$
\nabla^{2} V=\frac{\partial T}{\partial z}
$$

where $R_{t}=\alpha g \Delta T d^{3} / \nu \kappa$ and $R_{e a}=\gamma^{2} \varepsilon_{0} E_{0}^{2}(\Delta T)^{2} d^{2} / \mu \kappa$ are the thermal and AC electric Rayleigh numbers, respectively, $D a=k / d^{2}$ is the Darcy number, $T a=4 \Omega^{2} d^{4} / v^{2} \phi^{2}$ is the modified Taylor number, $\operatorname{Pr}=\nu \phi / \kappa$ is the Prandtl number and $\Lambda=\tilde{\mu} / \mu$ is the ratio of viscosities.

The isothermal boundaries of the porous layer are assumed to be either free or rigid and we have considered the following boundary conditions [1, 18]:

$$
w=\frac{\partial^{2} w}{\partial z^{2}}=T=\frac{\partial \xi}{\partial z}=\frac{\partial V}{\partial z}=0
$$

on the stress-free boundary and

$$
w=\frac{\partial w}{\partial z}=T=\xi=V=0
$$

on the rigid boundary.

\section{Linear stability analysis}

To carry out the linear stability analysis, we employ the normal mode analysis procedure in which we look for the solution of the form

$$
(w, T, V, \xi)=(W, \Theta, \Phi, Z)(z) \exp (i \ell x+i m y+\omega t)
$$

where $\ell$ and $m$ are the horizontal wave numbers in the $x$ and $y$ directions respectively and $\omega\left(=\omega_{r}+i \omega_{i}\right)$ is the growth rate. Substituting Eq.(29) in Eqs.(23) - (26), we obtain

$$
\begin{aligned}
& \left\{\frac{\omega}{P r}-\Lambda\left(D^{2}-a^{2}\right)+D a^{-1}\right\}\left(D^{2}-a^{2}\right) W=-R_{t} a^{2} \Theta-T a^{1 / 2} D Z-R_{e a} a^{2}(\Theta-D \Phi) \\
& \left\{\frac{\omega}{P r}-\Lambda\left(D^{2}-a^{2}\right)+D a^{-1}\right\} Z=T a^{1 / 2} D W \\
& \left\{A \omega-\left(D^{2}-a^{2}\right)\right\} \Theta=W \\
& \left(D^{2}-a^{2}\right) \Phi=D \Theta
\end{aligned}
$$

where $D=d / d z$ and $a=\sqrt{\ell^{2}+m^{2}}$ is the overall horizontal wave number.

On using Eq.(29) in the boundary conditions (27) and (28), we get respectively

$$
W=D^{2} W=\Theta=D Z=D \Phi=0
$$

and

$$
W=D W=\Theta=Z=\Phi=0
$$


The above set of equations is a double eigenvalue problem for $R_{t}$ or $R_{e a}$ and $\omega$, to be solved with respect to the chosen boundary conditions. Three types of velocity boundary conditions are considered for discussion namely, the boundaries are either (i) stress-free or (ii) rigid or (iii) lower boundary rigid and upper boundary stress-free.

\subsection{Exact solution for free-free boundaries}

For both boundaries stress-free, let us assume the solution in the following form such that they satisfy the respective boundary conditions:

$$
W=A_{1} \sin \pi z, \Theta=A_{2} \sin \pi z, Z=A_{3} \cos \pi z, \Phi=A_{4} \cos \pi z
$$

where $A_{1}-A_{4}$ are constants. Substituting Eq. (36) into Eqs. (30)-(33), we find the condition for the existence of a non-trivial eigenvalue is

$$
\left|\begin{array}{cccc}
\left(\frac{\omega}{P r}+\Lambda \delta^{2}+D a^{-1}\right) \delta^{2} & -\left(R_{t}+R_{e a}\right) a^{2} & \pi T a^{1 / 2} & -R_{e a} a^{2} \pi \\
-\pi T a^{1 / 2} & 0 & \left(\frac{\omega}{P r}+\Lambda \delta^{2}+D a^{-1}\right) \delta^{2} & 0 \\
-1 & 0 & A \omega+\delta^{2} & 0 \\
0 & \pi & 0 & \delta^{2}
\end{array}\right|=0
$$

where $\delta^{2}=\pi^{2}+a^{2}$. Expanding the above determinant yields the following cubic dispersion relation

$$
\omega^{3}+\delta_{1} \omega^{2}+\delta_{2} \omega+\delta_{3}=0
$$

where

$$
\begin{aligned}
& \delta_{1}=\delta^{2}\left(2 \operatorname{Pr} \eta+\frac{1}{A}\right) \\
& \delta_{2}=\frac{\operatorname{Pra}^{2}}{\delta^{2}}\left(\frac{\delta^{6} P r \eta^{2}}{a^{2}}+\frac{2 \delta^{6} \eta}{A a^{2}}+\frac{\pi^{2} P r T a}{a^{2}}-\frac{R_{t}}{A}-\frac{R_{e a} a^{2}}{\delta^{2} A}\right) \\
& \delta_{3}=\operatorname{Pr}^{2} a^{2} \eta\left(\frac{\delta^{6} \eta}{A a^{2}}+\frac{\pi^{2} T a}{a^{2} A \eta}-\frac{R_{t}}{A}-\frac{R_{e a} a^{2}}{A \delta^{2}}\right)
\end{aligned}
$$

with $\eta=\Lambda+D a^{-1} / \delta^{2}$. Putting $\omega=0$ in Eq. (38) and simplifying, it is found that stationary convection occurs at $R_{t}=R_{t}^{s}$, where

$$
R_{t}^{s}=\frac{T a \pi^{2}}{a^{2} \eta}+\frac{\delta^{6} \eta}{a^{2}}-\frac{R_{e a} a^{2}}{\delta^{2}} .
$$


It is interesting to check Eq.(40) for existing results in the literature under some limiting cases. In the absence of electric field (i.e., $R_{e a}=0$ ) and rotation (i.e., $T a=0$ ), the above equation reduces to

$$
R_{t}^{s}=\frac{\Lambda \delta^{6}+D a^{-1} \delta^{4}}{a^{2}}
$$

and this is the known result for Brinkman porous medium. We note that $R_{t}^{s}$ given by Eq. (40a) attains its critical value at $a=a_{c}$, where $a_{c}$ is the critical wave number given by

$$
a_{c}=\sqrt{\left\{\frac{-\left(\pi^{2} \Lambda+D a^{-1}\right)+\sqrt{\left(\pi^{2} \Lambda+D a^{-1}\right)\left(9 \pi^{2} \Lambda+D a^{-1}\right)}}{4 \Lambda}\right\}} \text {. }
$$

As $D a \rightarrow \infty$ and $\Lambda=1$ (viscous case), from (40b), we note that

$$
a_{c}=\pi / \sqrt{2}
$$

and the corresponding critical Rayleigh number $R_{t c}^{S}$, from (40a) is found to be

$$
R_{t c}^{S}=\frac{27 \pi^{4}}{4}
$$

which are the known exact values[1]. When $R_{e a}=0, \Lambda=1$ and as $D a \rightarrow \infty$, Eq. (40) reduces to

$$
R_{t}^{s}=\frac{T a \pi^{2}}{a^{2}}+\frac{\delta^{6}}{a^{2}}
$$

and coincides with Chandrasekhar [1]. When $T a=0, \Lambda=1$ and as $D a \rightarrow \infty$, Eq. (40) reduces to

$$
R_{t}^{s}=-\frac{R_{e a} a^{2}}{\delta^{2}}+\frac{\delta^{6}}{a^{2}}
$$

and coincides with Roberts [27].

To find the critical value of $R_{t}^{s}$, Eq. (40) is differentiated with respect to $a^{2}$ and equated to zero. A polynomial in $\left(a_{c}^{2}\right)$, whose coefficients are functions of the physical parameters influencing the instability is obtained in the form

$$
a_{7}\left(a_{c}^{2}\right)^{7}+a_{6}\left(a_{c}^{2}\right)^{6}+a_{5}\left(a_{c}^{2}\right)^{5}+a_{4}\left(a_{c}^{2}\right)^{4}+a_{3}\left(a_{c}^{2}\right)^{3}-a_{2}\left(a_{c}^{2}\right)^{2}-a_{1}\left(a_{c}^{2}\right)-a_{0}=0 .
$$

Where

$$
\begin{aligned}
& a_{7}=2 \Lambda^{3} \\
& a_{6}=11 \pi^{2} \Lambda^{3}+5 D a^{-1} \Lambda^{2}
\end{aligned}
$$




$$
\begin{aligned}
& a_{5}=24 \pi^{4} \Lambda^{3}+22 D a^{-1} \pi^{2} \Lambda^{2}+4\left(D a^{-1}\right)^{2} \Lambda \\
& a_{4}=25 \pi^{6} \Lambda^{3}-\pi^{2} R_{e a} \Lambda^{2}+35 D a^{-1} \pi^{4} \Lambda^{2}+13\left(D a^{-1}\right)^{2} \pi^{2}-\pi^{2} T a \Lambda+\left(D a^{-1}\right)^{3} \\
& a_{3}=2\left(D a^{-1}\right)^{3} \pi^{2}+12\left(D a^{-1}\right)^{2} \pi^{4} \Lambda-2 D a^{-1} \pi^{2} R_{e a} \Lambda-4 \pi^{4} T a \Lambda+20 D a^{-1} \pi^{6} \Lambda^{2} \\
& -2 \pi^{4} R_{e a} \Lambda^{2}+10 \pi^{8} \Lambda^{3} \\
& a_{2}=\left(D a^{-1}\right)^{2} \pi^{2} R_{t}+D a^{-1} \pi^{4} T a+2\left(D a^{-1}\right)^{2} \pi^{6} T a+2 D a^{-1} \pi^{4} R_{e a} \Lambda+6 \pi^{6} T a \Lambda \\
& +5 D a^{-1} \pi^{8} \Lambda^{2}+\pi^{6} R_{e a} \Lambda^{2}+3 \pi^{10} \Lambda^{3} \\
& a_{1}=2\left(D a^{-1}\right)^{3} \pi^{6}+2 D a^{-1} \pi^{6} T a+8\left(D a^{-1}\right)^{2} \pi^{8} \Lambda+4 \pi^{8} T a \Lambda+10 D a^{-1} \pi^{10} \Lambda^{2}+4 \pi^{12} \Lambda^{3} \\
& a_{0}=\left(D a^{-1}\right)^{3} \pi^{8}+\left(D a^{-1}\right)^{3} \pi^{8} T a+3\left(D a^{-1}\right)^{2} \pi^{10} \Lambda+\pi^{10} \Lambda T a+3 D a^{-1} \pi^{12} \Lambda^{2}+\pi^{4} \Lambda^{3} .
\end{aligned}
$$

The above equation is solved numerically for various values of $R_{e a}, T a, \Lambda$ and $D a^{-1}$ and the minimum value of $a_{c}^{2}$ is obtained each time, hence the critical wave number is obtained. Using this in Eq. (40), the critical Rayleigh number, above which the convection sets- in is determined.

In the absence of electric field ( $\left.R_{e a}=0\right)$, Eq. (41) reduces to

$$
\begin{aligned}
2\left(a_{c}^{2}\right)^{5}+5\left(\eta^{\prime}+\right. & \left.0.4 \pi^{2}\right)\left(a_{c}^{2}\right)^{4}+4 \eta^{\prime}\left(\eta^{\prime}+\pi^{2}\right)\left(a_{c}^{2}\right)^{3}+\left\{\eta^{\prime 2}+2 \pi^{2} \eta^{\prime}-\pi^{4}-\frac{\pi^{2} T a}{\Lambda}\right\}\left(a_{c}^{2}\right)^{2} \\
- & \pi^{4}\left(\eta^{\prime 2}+\frac{T a}{\Lambda^{2}}\right)\left(2\left(a_{c}^{2}\right)+\eta^{\prime}\right)=0
\end{aligned}
$$

where $\eta^{\prime}=\pi^{2}+D a^{-1} / \Lambda$.

When $D a \rightarrow \infty$ and $\Lambda=1$ (non-porous domain case), Eq. (42) can be written as

$$
\left\{2\left(a_{c}^{2}\right)^{3}+3 \pi^{2}\left(a_{c}^{2}\right)^{2}-\pi^{6}\left(1+\frac{T a}{\pi^{4}}\right)\right\}\left(\left(a_{c}^{2}\right)+\pi^{2}\right)^{2}=0 .
$$

Since $\left(a_{c}^{2}+\pi^{2}\right)^{2} \neq 0$, it follows that

$$
2\left(a_{c}^{2}\right)^{3}+3 \pi^{2}\left(a_{c}^{2}\right)^{2}-\pi^{6}\left(1+\frac{T a}{\pi^{4}}\right)=0
$$

a result which coincides with Chandrasekhar [1].

To analyze the onset of oscillatory convection, we substitute $\omega=i \omega_{i}$, where $\omega_{i}$ is a nonzero real number, in Eq. (36) and equate the real and imaginary parts of the corresponding equation to obtain 


$$
\omega_{i}^{2}=\frac{\delta_{3}}{\delta_{1}} \text { and } \omega_{i}^{2}=\delta_{2}
$$

Eliminating $\omega_{i}$ finally leads to an expression for the Rayleigh number $R_{t}=R_{t}^{0}$ at which the oscillatory convection occurs, where

$$
R_{t}^{0}=\frac{2 \eta(1+\operatorname{Pr} A \eta)}{a^{2}}\left[\delta^{6}+\pi^{2} \operatorname{Ta}\left(\frac{A P r}{1+\operatorname{Pr} A \eta}\right)^{2}\right]-\frac{R_{e a} a^{2}}{\delta^{2}}
$$

Now the condition that $\omega_{i}^{2}>0$ provides the fact that oscillatory instability can occur only if

$$
0<\operatorname{Pr}<\frac{1}{A \eta} \quad \text { and } \quad \operatorname{Ta}>\frac{\delta^{6} \eta^{2}(1+\operatorname{Pr} A \eta)}{\pi^{2}(1-\operatorname{Pr} A \eta)} .
$$

It is interesting to note that these conditions are exactly in the same form as that of the ordinary viscous fluid saturating a rotating Brinkman porous layer (Shivakumara et al. [11]) suggesting that the vertical AC electric field does not influence the necessary conditions for the existence of oscillatory convection. If $T a=0$, then we note that the instability sets in only via stationary convection. Further, the value of $\operatorname{Pr}$ depends on the physical parameters $D a, \Lambda$, $\phi$ and $A$ also. For the non-porous case (i.e., $D a \rightarrow \infty, \Lambda=1$ and $A=1=\phi$ ), the above conditions become

$$
\operatorname{Pr}<1, \quad \text { Ta }>\frac{\delta^{6}(1+P r)}{\pi^{2}(1-P r)}
$$

and coincide with those given by Chandrasekhar [1]. A glance at Eq. (47) reveals that the onset of oscillatory convection in a rotating porous layer is not limited to a particular domain of Prandtl number values as in the case of pure fluids. Moreover, it is interesting to note that $\omega_{i}^{2}$ can be written in the form

$$
\omega_{i}^{2}=\frac{\operatorname{Pr}^{2} \eta a^{2}}{\delta^{2}(1+2 \operatorname{Pr} \eta A)}\left(R_{t}^{s}-R_{t}^{0}\right) .
$$

From Eq. (49) it is evident that if the instability sets in as oscillatory motions, it always occurs at a Rayleigh number less than the stationary onset.

\subsection{Numerical solution for rigid-rigid and rigid-free boundaries}

It has been observed that oscillatory convection occurs only if the Prandtl number $\mathrm{Pr}$ is less than unity and the Taylor number exceeds a threshold. But for dielectric liquids, 
Prandtl number is much greater than unity (for example, for corn oil $\operatorname{Pr}=480$, silicone oil $\operatorname{Pr}=100$ and for caster oil $\operatorname{Pr}=10000)$ and hence the oscillatory convection is ruled out as the preferred mode of instability. Under the circumstances, we restrict ourselves to the case of steady onset and put $\omega=0$ in Eqs. (27) - (30). As in the case of stress-free boundaries, an exact solution is not possible for these two types of boundary conditions and the Galerkin method as explained in the book by Finlayson [28] is adopted to solve the resulting eigenvalue problem. Accordingly, the variables are written in a series of basis functions as

$$
W=\sum A_{i} W_{i}, \Theta=\sum B_{i} \Theta_{i}, Z=\sum C_{i} Z_{i}, \Phi=\sum D_{i} \Phi_{i}
$$

where $A_{i}, B_{i}, C_{i}$ and $D_{i}$ are constants and the basis functions $W_{i}, \Theta_{i}, Z_{i}$ and $\Phi_{i}$ will be represented by the power series satisfying the respective boundary conditions. Substituting (50) into (50)-(53), multiplying the resulting momentum equation by $W_{j}(z)$, vorticity equation by $\xi_{j}(z)$, energy equation by $\Theta_{j}(z)$, electric potential equation by $\Phi_{j}(z)$; performing the integration by parts with respect to $\mathrm{z}$ between $\mathrm{z}=0$ and $\mathrm{z}=1$ and using the boundary conditions, leads to the following system of linear homogeneous algebraic equations:

$$
\begin{aligned}
& E_{j i} A_{i}+F_{j i} B_{i}+G_{j i} C_{i}+H_{j i} D_{i}=0 \\
& I_{j i} A_{i}+J_{j i} B_{i}=0 \\
& K_{j i} A_{i}+L_{j i} C_{i}=0 \\
& M_{j i} B_{i}+N_{j i} D_{i}=0
\end{aligned}
$$

where

$$
\begin{aligned}
& E_{j i}=\Lambda<D^{2} W_{j} D^{2} W_{i}+a^{4} W_{j} W_{i}+2 a^{2} D W_{j} D W_{i}>+D a^{-1}<D W_{j} D W_{i}+a^{2} W_{j} W_{i}> \\
& F_{j i}=-<R_{t} a^{2} W_{j} \Theta_{i}+R_{e a} a^{2} W_{j} \Theta_{i}>, G_{j i}=-<T a^{1 / 2} W_{j} D Z_{i}>, H_{j i}=<R_{e a} a^{2} W_{j} D \Phi_{i}> \\
& I_{j i}=<\Theta_{j} W_{i}>, J_{j i}=-<D \Theta_{j} D \Theta_{i}+a^{2} \Theta_{j} \Theta_{i}>, K_{j i}=<T a^{1 / 2} Z_{j} D W_{i}> \\
& L_{j i}=-<\Lambda\left(D Z_{j} D Z_{i}+a^{2} Z_{j} Z_{i}\right)+D a^{-1} Z_{j} Z_{i}>, M_{j i}=-<\Phi_{j} D \Theta_{i}> \\
& N_{j i}=-<D \Phi_{j} D \Phi_{i}+a^{2} \Phi_{j} \Phi_{i}>.
\end{aligned}
$$

Here the inner product is defined as $\langle f g\rangle=\int_{0}^{1} f g d z$.

The above set of homogeneous algebraic equations can have a non-trivial solution if and only if 


$$
\left|\begin{array}{llll}
E_{j i} & F_{j i} & G_{j i} & H_{j i} \\
I_{j i} & J_{j i} & 0 & 0 \\
K_{j i} & 0 & L_{j i} & 0 \\
0 & M_{j i} & 0 & N_{j i}
\end{array}\right|=0 .
$$

We select trial functions satisfying the appropriate boundary conditions. For both boundaries rigid case, the trial functions chosen are

$$
W_{i}=z^{i+1}-2 z^{i+2}+z^{i+3}, \Theta_{i}=z^{i}-z^{i+1}, \Phi_{i}=z^{i}-z^{i+1}, Z_{i}=z^{i}-z^{i+1}
$$

while the trial functions chosen in the case of lower boundary rigid and upper boundary free are

$$
W_{i}=2 z^{i+3}+3 z^{i+1}-5 z^{i+2}, \Theta_{i}=z^{i}-z^{i+1}, Z_{i}=z^{i+2}-z^{i+1}-z^{i}, \Phi_{i}=z^{i+2}-z^{i+1}-z^{i} .
$$

Substituting Eq. (53) or (54) as the case may be in Eq.(52) and expanding the determinant leads to the characteristic equation giving the thermal Rayleigh number $R_{t}$ as a function of the wave number $a$ as well as other parameters $D a, R_{e a}$, Ta and $\Lambda$. The inner products involved in the determinant are evaluated analytically rather than numerically in order to avoid errors in the numerical integration. The critical Rayleigh number $R_{t c}$ is obtained by minimizing $R_{t}$ with respect to the wave number $a$ for different fixed values of other parameters. Computations reveal that the convergence in finding $R_{t c}$ crucially depends on the value of $T a$, and for higher values of $T a$ more number of terms are found to be required in the Galerkin expansion. The results presented here are for $i=j=8$ the order at which the convergence is achieved, in general.

\section{Results and discussion}

The simultaneous effect of Coriolis force and vertical AC electric field on the criterion for the onset of convection in a dielectric fluid saturated rotating Brinkman porous layer heated from below is investigated. Attention is focused on three types of velocity boundary conditions namely, (i) both boundaries free, (ii) both boundaries rigid and (iii) lower boundary rigid and upper boundary free. To solve the resulting eigenvalue problem, both analytical and numerical techniques are used depending on the choice of velocity boundary conditions. Since the oscillatory convection is not a preferred mode of instability for the problem considered, the discussion is limited to stationary onset. 


\subsection{Both boundaries stress-free}

From Eq. (40), we note $\partial R_{t}^{s} / \partial T a>0$ and $\partial R_{t}^{s} / \partial R_{e a}<0$ indicating that $R_{t}^{s}$ is an increasing function of $\mathrm{Ta}$ but decreasing function of $R_{e a}$ and hence the effect of increasing $T a$ and $R_{e a}$ is to delay and hasten the onset of electrothermal convection, respectively. Nonetheless, it is noted that

$$
\frac{\partial R_{t}^{s}}{\partial D a^{-1}}=\frac{\delta^{4}}{a^{2}}-\frac{\pi^{2} T a}{a^{2}\left(\Lambda+\frac{D a^{-1}}{\delta^{2}}\right)^{2} \delta^{2}}
$$

and

$$
\frac{\partial R_{t}^{s}}{\partial \Lambda}=\frac{\delta^{6}}{a^{2}}-\frac{\pi^{2} T a}{a^{2}\left(\Lambda+\frac{D a^{-1}}{\delta^{2}}\right)^{2}} .
$$

Here, it is observed that the right-hand side of the above equations may be either negative or positive depending on the parametric values. That is to say that an increase in the value of $D a^{-1}$ and $\Lambda$ might lead to an instability of a rotating porous layer in the presence of an $\mathrm{AC}$ electric field; a contrast result noted when compared to non-rotating case. Distinctly this fact has been exhibited graphically in Figs. 2 and 3.

Figure 2 depicts the curves of $R_{t c}$ as a function of $D a^{-1}$ for different values of $\Lambda(=1$, $2,3$ and 4$)$ and $R_{e a}(=0,500,1000)$ when the value of $\mathrm{Ta}$ fixed at $10^{5}$. From the figure it is seen that the destabilization due to $D a^{-1}$ manifests itself as minimum in the $R_{t c}-D a^{-1}$ curve. The range of $\mathrm{Da}^{-1}$ up to which the system becomes destabilized increases with the decrease in the value of $\Lambda$. This may be due to a delicate balance between Coriolis and Darcy frictional forces, while elsewhere a strong 'two-dimensionality prevails, being provided at lower values of $D a^{-1}$ by Coriolis forces, and at higher values of $D a^{-1}$ by frictional forces. This phenomenon is similar to the one observed by Chandrasekhar [1] in the study of thermal instability in a rotating fluid layer in the presence of vertical magnetic field; where it is observed that the simultaneous presence of rotation and magnetic field destabilizes the system although their individual effect is to make the system more stable. Moreover, it is found that $R_{t c}$ attains its minimum value with $D a^{-1}$, denoted by $\left(R_{t c}\right)_{\min }$, at $D a^{-1}=D a_{m}^{-1}$, where 


$$
D a_{m}^{-1}=\frac{\pi \sqrt{T a} \sqrt{\pi^{2}+a_{c}^{2}}-a_{c}^{4} \Lambda\left(\pi^{4}+1\right)-2 \pi^{2} a_{c}^{2} \Lambda}{\pi^{2}+a_{c}^{2}} .
$$

It is evident that $D a_{m}^{-1}$ decreases with an increase in the value of $\Lambda$ but increases with an increase in the value of $\mathrm{Ta}$.

A similar type of behavior, as observed above, is noticed when we allow to vary $\Lambda$ by fixing $D a^{-1}$ and the results are presented in Fig. 3. The dual role of viscosity ratio $\Lambda$ on the stability of a rotating porous layer is evident from Fig. 3. It is observed that $R_{t c}$ attains its minimum value in the $R_{t c}-\Lambda$ plane. It is noted that the system gets destabilized up to a certain value of $\Lambda$ and the range of this value goes on decreasing with increasing $D a^{-1}$. Here, $\left(R_{t c}\right)_{\min }$ denotes the minimum value attained by $R_{t c}$ with respect to $\Lambda$ when the other parameters are held fixed. In this case, $R_{t c}$ attains its minimum value with $\Lambda$ (i.e., $\left.\left(R_{t c}\right)_{\min }\right)$ at $\Lambda=\Lambda_{\min }$, where

$$
\Lambda_{\min }=\frac{-D a^{-1}}{\left(\pi^{2}+a_{c}^{2}\right)}+\frac{\pi \sqrt{T a}}{\left(\pi^{2}+a_{c}^{2}\right) \sqrt{\left(\pi^{2}+a_{c}^{2}\right)}} .
$$

It is seen that $\Lambda_{\text {min }}$ decreases with an increase in the value of $D a^{-1}$, while it increases with increase in $T a$. In this case the coupling between $D a^{-1}$ and $\Lambda_{\min }$ is found such that $\left(R_{t c}\right)_{\min }$ value remains unaltered and coincides with the one obtained previously by varying $\Lambda$.

\subsection{Both boundaries rigid, and lower rigid and upper stress-free boundaries}

For these two boundary combinations, the eigenvalue problem is solved numerically using the Galerkin technique. To validate the numerical procedure used, first the test computations are carried out under different limiting cases for these two boundary conditions. The critical Rayleigh number $R_{t c}$ and the corresponding wave number $a_{c}$ obtained for different values of $T a$ when $R_{e a}=0, D a^{-1}=0$ and $\Lambda=1$ (i.e., classical viscous case) are compared with those of Chandrasekhar [1] in Table 1 for rigid-rigid boundaries, while the critical AC electric Rayleigh number $R_{e a c}$ and the corresponding wave number obtained for rigid boundaries for different values of $R_{t}$ when $T a=0$ are compared with those of Roberts [27] in Table 2. From these Tables it is seen that the results obtained from the present solution procedure are in excellent agreement with the earlier published ones and thus verifies the accuracy of the method used. 
As observed in the stress-free boundaries case, the numerically computed results reveal that both $D a^{-1}$ and $\Lambda$ show some destabilization on the onset of stationary convection even in the case of rigid-rigid and rigid-free boundaries. Figures (4) and (5) depict this aspect for rigid-rigid and rigid-free boundaries respectively in the $R_{t c}-\Lambda$ plane for different values of $D a^{-1}$. From these two figures it is seen that $R_{t c}$ passes through a minimum with an increase in the value of $\Lambda$ and the crossing of curves shows the destabilization due to $D a^{-1}$ as well. Further, the range of $\Lambda$ up to which the system gets destabilized decreases with increasing $\mathrm{Da}^{-1}$ as observed in the case of free-free boundaries. The destabilization due to increasing $D a^{-1}$ is shown in Figs. 6 and 7 for different values of $\Lambda$ for rigid-rigid and rigidfree boundaries respectively. The destabilization manifests itself as minimum in the $R_{t c}-D a^{-1}$ plane. From the figures it is noted that the range of $D a^{-1}$ up to which the system gets destabilized increases with the decrease in the value of $\Lambda$.

\subsection{Comparison of results for different types of boundaries}

The critical thermal Rayleigh number $R_{t c}$ and the corresponding wave number $a_{c}$, computed for different values of physical parameters and velocity boundary conditions, are illustrated in Figs. 8(a,b)-10(a,b) and also tabulated in Tables 3-6 with the perspective of understanding the effect of boundaries on the onset of electrothermoconvection in a rotating porous layer. The variation of $R_{t c}$ and $a_{c}$ is presented in Figs. 8(a) and 8 (b), respectively as

a function of $R_{e a}$ for different values of $D a^{-1}$ with $T a=1000$ and $\Lambda=4$. Figure 8(a) shows that the onset of electrothermoconvection is delayed with increasing $D a^{-1}$. This is because of decrease in the permeability of the porous medium, which in turn requires more heating for instability. On the contrary, when the AC electric field is increased, the porous layer becomes more and more unstable due to an increase in the electrical destabilizing effect. In other words, the effect of increasing AC electric field strength is to enhance the heat transfer and to hasten the onset of convection. We note that the results for different velocity boundary conditions differ only quantitatively, and the system is found to be more stable when both boundaries are rigid, while the free boundaries are the least stable for the moderate values of Taylor number considered. This figure also exhibits that the deviation in the $R_{t c}$ values between different boundary conditions increases slightly with an increase in the value of $D a^{-1}$. Figure 8(b) illustrates that the critical wave number $a_{c}$ increases slowly with an 
increase in the value of $R_{e a}$ but significantly increases with an increase in the value of $D a^{-1}$ in the case of free-free and rigid-free boundaries when compared to rigid-rigid boundaries. Thus their effect is to contract the size of convection cells. Further, it is observed that

$$
\left(a_{c}\right)_{\text {rigid-rigid }}>\left(a_{c}\right)_{\text {rigid-free }}>\left(a_{c}\right)_{\text {free-free }} \text {. }
$$

Figures 9(a) and 9(b) respectively show the variation of $R_{t c}$ and $a_{c}$ as a function of $R_{e a}$ for several values of Taylor number with $D a^{-1}$ fixed to 100 and $\Lambda$ fixed to 4 . The results indicate that an increase in the strength of rotation is to delay the onset of electrothermo convection. Figure 9(b) depicts that the critical wave number increases with increasing $\mathrm{Ta}$ and hence its effect is to contract the size of convection cells.

As pointed out in the introduction, Brinkman's model rests on an effective viscosity $\tilde{\mu}$ different from fluid viscosity $\mu$ denoted through $\Lambda$ in dimensionless form and it has a determining influence on the onset of electrothermal convection in porous media. The influence of viscosity ratio on the critical stability parameters is summarized in Figs. 10(a) and $10(\mathrm{~b})$. It is seen that $R_{t c}$ as well as deviation in its values among different boundary conditions increases with an increase in the value of $\Lambda$ (see Fig. 10a). In contrast to this, the critical wave number decreases with $\Lambda$ (see Fig. 10b). Thus the effect of $\Lambda$ is to suppress the onset of electrothermal convection due to an increase in viscous diffusion and also to increase the dimension of convection cells.

The values of $\Lambda_{\min }, D a_{m}^{-1}$ and the corresponding $\left(R_{t c}\right)_{\min }$ computed numerically for different values of $D a^{-1}, \Lambda$ and $R_{e a}$ for free-free, rigid-rigid and rigid-free boundaries when $T a=10^{5}$ and $T a=2 \times 10^{5}$ are compared in Tables $3-6$. From the tables, it may be noted that there is a coupling between the values of $\Lambda$ and $D a_{m}^{-1}$ as well as $D a^{-1}$ and $\Lambda_{\text {min }}$ such that the values of $\left(R_{t c}\right)_{\min }$ remain the same for a fixed value of $R_{e a}$ in the case of free boundaries. In particular, it is observed that increasing $\Lambda$ and $R_{e a}$ is to decrease $D a_{m}^{-1}$, while increasing $T a$ is to increase $\left(R_{t c}\right)_{\min }$ as well as $D a_{m}^{-1}$. Nonetheless, no such coupling is found between either $D a^{-1}$ and $\Lambda_{\text {min }}$ or $\Lambda$ and $D a_{\text {min }}^{-1}$ such that $\left(R_{t c}\right)_{\min }$ remains same in the case of rigid-rigid and rigid-free boundaries. Besides, the range of $\Lambda$ up to which the system gets destabilized is the least for rigid-rigid boundaries and the range is more in the case of free-free boundaries. The stress-free boundaries are found to be less stable than rigid-free as well as rigid-rigid boundaries only at small and moderate values of Taylor number domain (see Fig. 9a). To the 
contrary, the stress-free boundaries are found to be more stable than that of rigid-rigid and rigid-free boundaries at higher values of Taylor number. This is because a viscous boundary layer will appear near the rigid boundary which in turn arrests the fluid motion. Although the boundary layer exerts a pure stabilizing mechanism up to moderate values ofTa, it exhibits a dual effect at higher values of Ta. On the one hand, the viscosity dissipates the kinetic energy required for the onset of instability in the porous layer; on the other hand, the viscous force resists the fluid to attach to the vortex lines and makes the fluid to find a means for achieving cross-isobar flow through which potential energy is released [21]. The dual mechanisms coupled with the EHD force exhibit a more instability behavior in the case of rigid boundary.

\section{Conclusions}

The effect of Coriolis force due to rotation on the onset of electrothermal convection in a dielectric fluid saturated porous medium has been investigated. The analysis has been performed for three different types of velocity boundary conditions namely free-free, rigidrigid and lower-rigid and upper-free boundaries. It is observed that both rotation and vertical AC electric field play a vital role on the stability characteristics of the system. Dual behavior of porous medium permeability and ratio of viscosities on the criterion for the onset of electrothermoconvection is observed in the presence of rotation. Under some choices of parametric values, it is found that decrease in the Darcy number and increase in the ratio of viscosities destabilize the system contrary to their stabilizing effect in the absence of rotation.

The range of $D a^{-1}$ and $\Lambda$ up to which the system becomes destabilized increases with the decrease in the value of $\Lambda$, and increase in $D a^{-1}$ respectively. Besides, the effect of increasing $\mathrm{AC}$ electric field enhances the heat transfer and hence hastens the onset of convection, while increasing the strength of rotation is to inhibit the onset of electrothermoconvection. The system is found to be more stable when both boundaries are rigid, while the free boundaries are least stable only up to moderate values of Taylor number. At higher Taylor number, the trend is reversed since the stability of the free-free boundaries will be enhanced rapidly than the rigid boundaries case. The effect of increase in $T a, R_{e a}$ and $D a^{-1}$ is to contract the dimension of the convection cells, while increase in $\Lambda$ is to elongate the same. 


\section{Acknowledgements}

One of the authors (ISS) wishes to thank The University of Hong Kong for inviting him as a visiting Professor and also the Bangalore University for sanctioning sabbatical leave. The work was supported by the Research Grants Council of the Hong Kong Special Administrative Region, China, through Project Nos. HKU 715609E and HKU 715510E.

\section{References}

[1] S. Chandrasekhar, Hydrodynamic and hydromagnetic stability, Oxford University Press, London, 1961.

[2] G.P. Galdi, B. Straughan, Nonlinear analysis of the stabilizing effect of rotation in the Benard problem, Proc. R. Soc. London A 402 (1985) 257-283.

[3] R.C. Kloosterziel, G.F. Carnevale, Closed form linear stability conditions for rotating Rayleigh-Benard convection with rigid stress- free upper and lower boundaries, J. Fluid Mech. 480 (2003) 25-42.

[4] P.R. Patil, G. Vaidyanathan, On setting up of convection currents in a rotating porous medium under the influence of variable viscosity, Int. J. Engng Sci. 21 (1983) 123130.

[5] E. Palm, P.A. Tyvand, Thermal convection in a rotating porous layer. J. Appl. Math. Phys. (ZAMP) 35 (1984) 122-133.

[6] Y. Qin, P.N. Kaloni, Nonlinear stability problem of a rotating porous layer. Q. J. Appl. Math. LIII (1995) 129-142.

[7] P. Vadasz, Coriolis effect on gravity-driven convection in a rotating porous layer heated from below. J. Fluid Mech. 276 (1998) 351-375.

[8] P. Vadasz, Flow and thermal convection in rotating porous media. In: Vafai, K. (ed.) Handbook of Porous Media,. Marcel Dekker, Inc, New York (2000), 395-440.

[9] B. Straughan, A sharp nonlinear stability threshold in rotating porous convection. Proc. R. Soc. London A 457 (2001) 87-93.

[10] T. Desaive, M. Hennenberg, G. Lebon, Thermal instability of a rotating saturated porous medium heated from below and submitted to rotation, Eur. Phys. J. B, 29 (2002) 641-647.

[11] I.S. Shivakumara, M.N. Savitha, Krishna B. Chavaraddi, N. Devaraju, Bifurcation analysis for thermal convection in a rotating porous layer. Meccanica 44 (2009) 225238. 
[12] P. Falsaperla, G. Mulone, B. Straughan, Rotating porous convection with prescribed heat flux, Int. J. Engng. Sci. 48 (2010) 685-692.

[13] T.B. Jones, Electrohydrodynamically enhanced heat transfer in liquids-A review in Advances in Heat Transfer. Ed. By T.F. Irvine Jr. and J.P. Hartnett. Academic Press, (1978) 107-144.

[14] D.A. Saville, Electrohydrodynamics: The Taylor-Melcher leaky dielectric model. Annu. Rev. Fluid Mech. 29 (1997) 27-64.

[15] I.S. Shivakumara, M.S. Nagashree, K. Hemalatha, Electroconvective instability in a heat generating dielectric fluid layer, Int. Comm. in Heat Mass Transf. 34 (2007) 1041-1047.

[16] I.S. Shivakumara, N. Rudraiah, K. Hemalatha, Electrothermoconvection in a dielectric fluid layer in the presence of heat generation, Int. J. Appl. Math. 1 (2009) 87-101.

[17] M. H. Chang, A. C. Ruo, and F. Chen, Electrohydrodynamic instability in a horizontal fluid layer with electrical conductivity gradient subject to a weak shear flow, J. Fluid Mech. 634 (2009) 191-215.

[18] M. Takashima, The effect of rotation on electrohydrodynamic instability, Can. J. Phys. 54 (1976) 342-347.

[19] A. Douiebe, M. Hannaoui, G. Lebon, A. Benaboud, A. Khmou, Effects of a.c. electric field and rotation on Benard-Marangoni convection, Flow, Turbulence and Combustion 67 (2001) 185-204.

[20] M.I. Othman, Electrohydrodynamic instability of a rotating layer of a viscoelastic fluid heated from below, ZAMP 55 (2004) 468-482.

[21] A.C. Ruo, M.H. Chang, F. Chen, Effect of rotation on the electrohydrodynamic instability of a fluid layer with an electrical conductivity gradient, Phys. Fluids 22 (2010) 024102-1- 024102-11.

[22] P.H. Roberts, Electrohydrodynamic convection, Quart. J. Mech. Appl. Math. 22 (1969) 211-220.

[23] N. Rudraiah, M.S. Gayathri, Effect of thermal modulation on the onset of electrothermoconvection in a dielectric fluid saturated porous medium, ASME J. Heat Tranf. 131 (2009) 101009-101015.

[24] S.C. Subramanian, K.R. Rajagopal, A note on the flow through porous solids at high pressures, Comput. Math. Appl. 53(2007) 260-275.

[25] K. Kannan, K. R. Rajagopal, Flow through porous media due to high pressure gradients, Appl. Math. Comput. 199 (2008) 748-759. 
[26] K. R. Rajagopal, G. Saccomandi, L. Vergori, A systematic approximation for the equations governing convection diffusion in a porous medium, Nonlinear Analysis: Real World Appl. 11 (2010) 2366-2375.

[27] R. Z. Moreno, E. J. Bonet, O.V. Trevisan,. Electric alternating current effects on flow of oil and water in porous media, in: Vafai, K and Shivakumar, P.N., (eds.). Proceedings of the International Conference on Porous Media and Their Applications in Science, Engineering and Industry, Hawaii. (1996) 147-172.

[28] B.A. Finlayson, The Method of Weighted Residuals and Variational Principles, Academic Press, 1972.

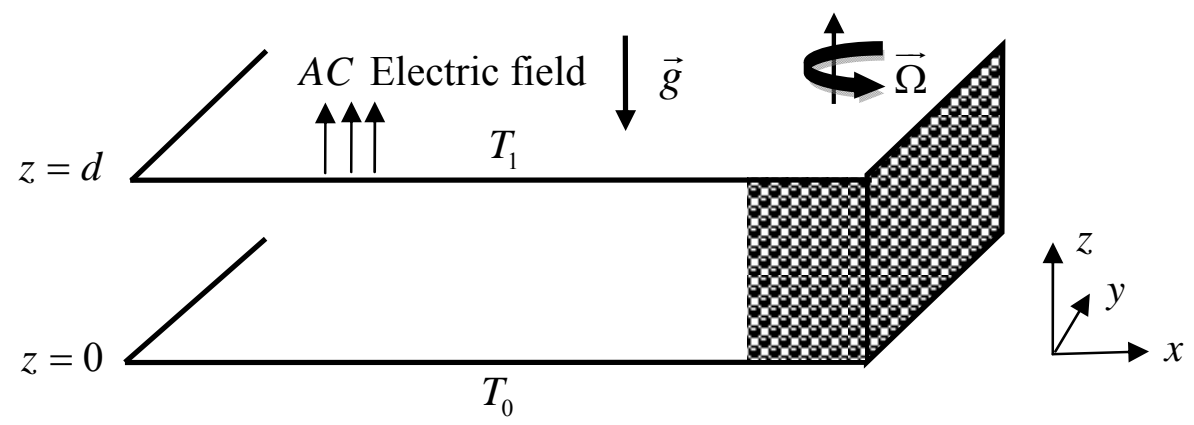

Fig. 1. Physical Configuration 


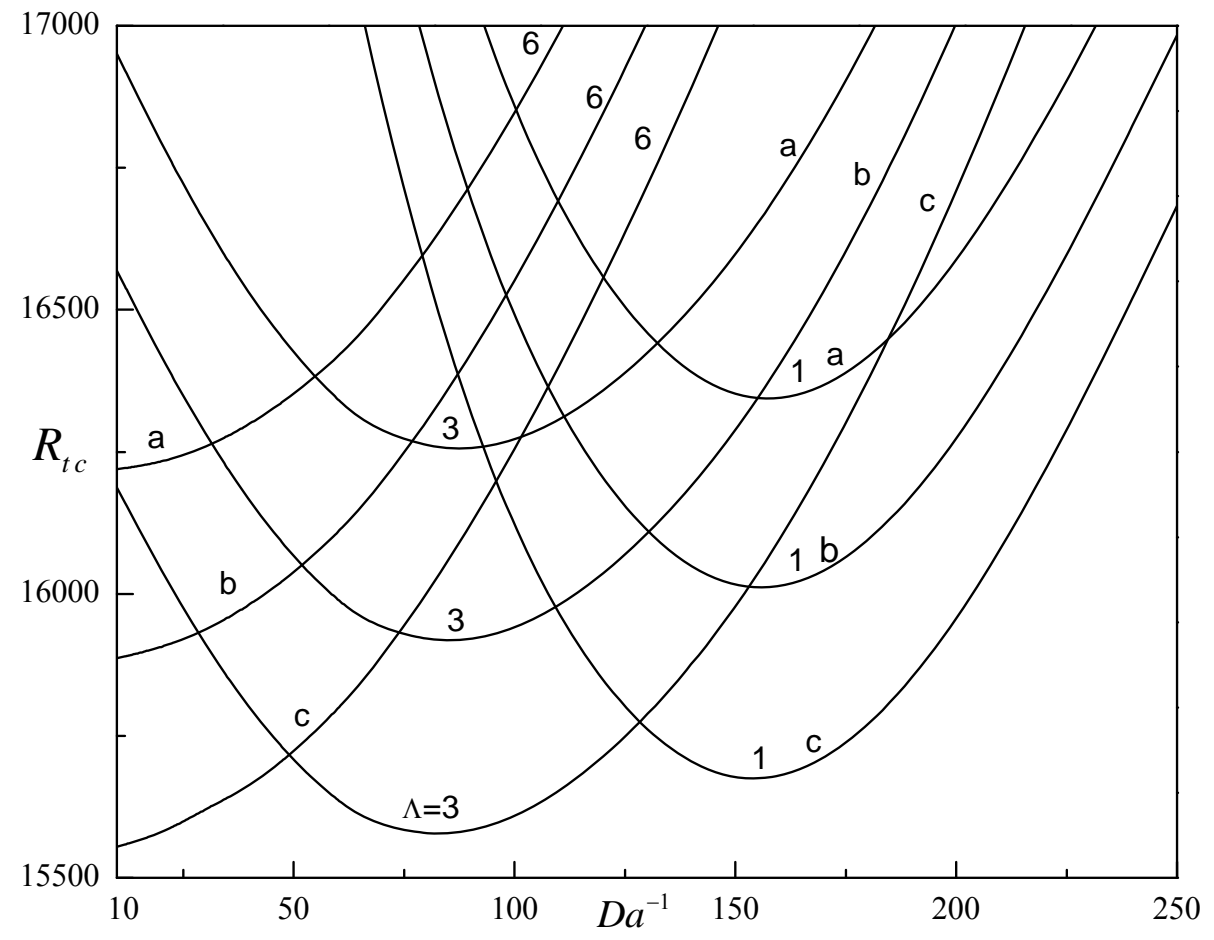

Fig. 2. $R_{t c} \operatorname{Vs} D a^{-1}$ for different values of $\Lambda$ and $R_{e a}$ when $T a=10^{5}$ for (a) $R_{e a}=0$, (b) $R_{e a}=500$ and (c) $R_{e a}=1000$ for free-free boundaries

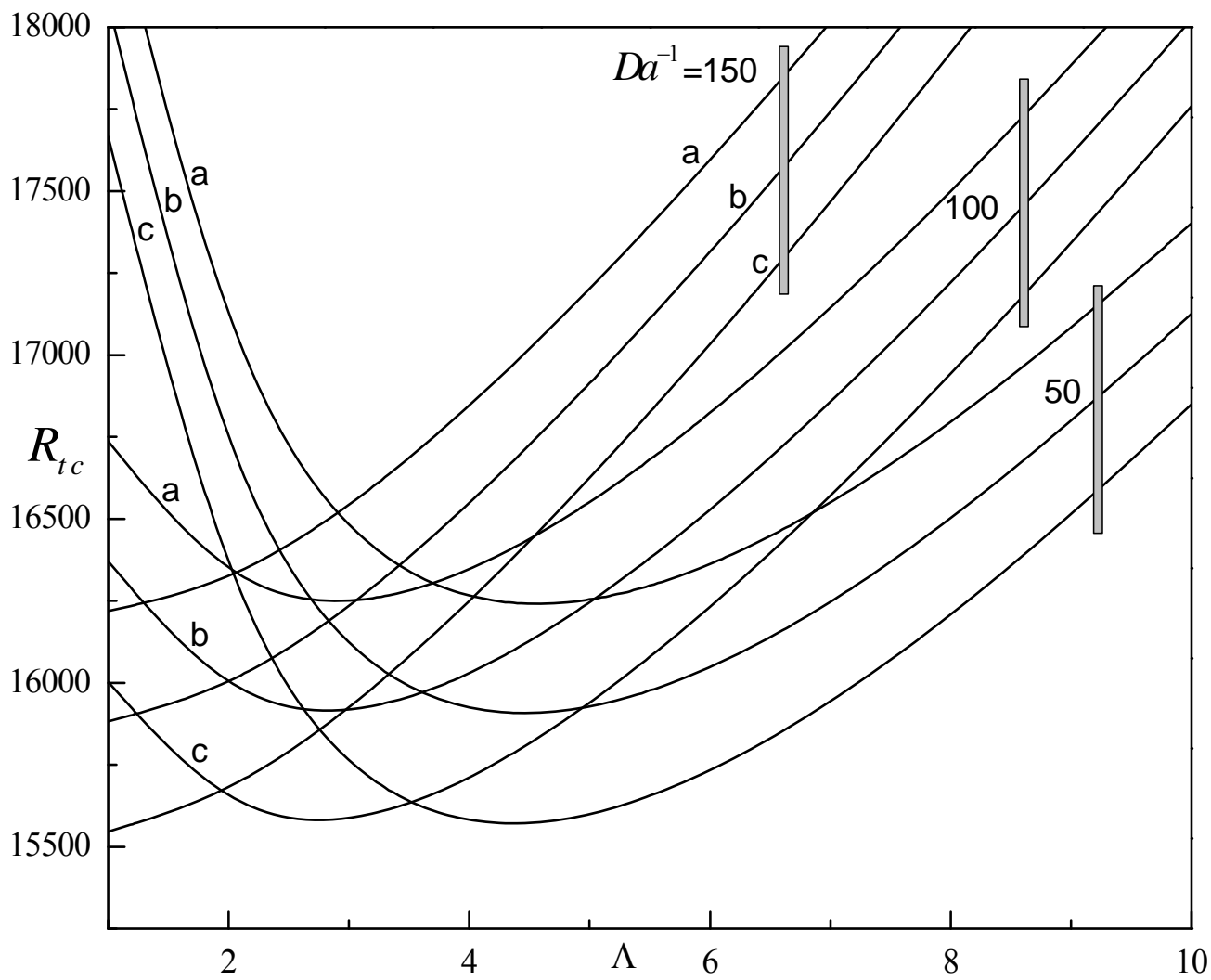

Fig. 3. $R_{t c} \operatorname{Vs} \Lambda$ for different values of $D a^{-1}$ and $R_{e a}$ when $T a=10^{5}$ for (a) $R_{e a}=0$, (b) $R_{e a}=500$ and (c) $R_{e a}=1000$ for free-free boundaries 


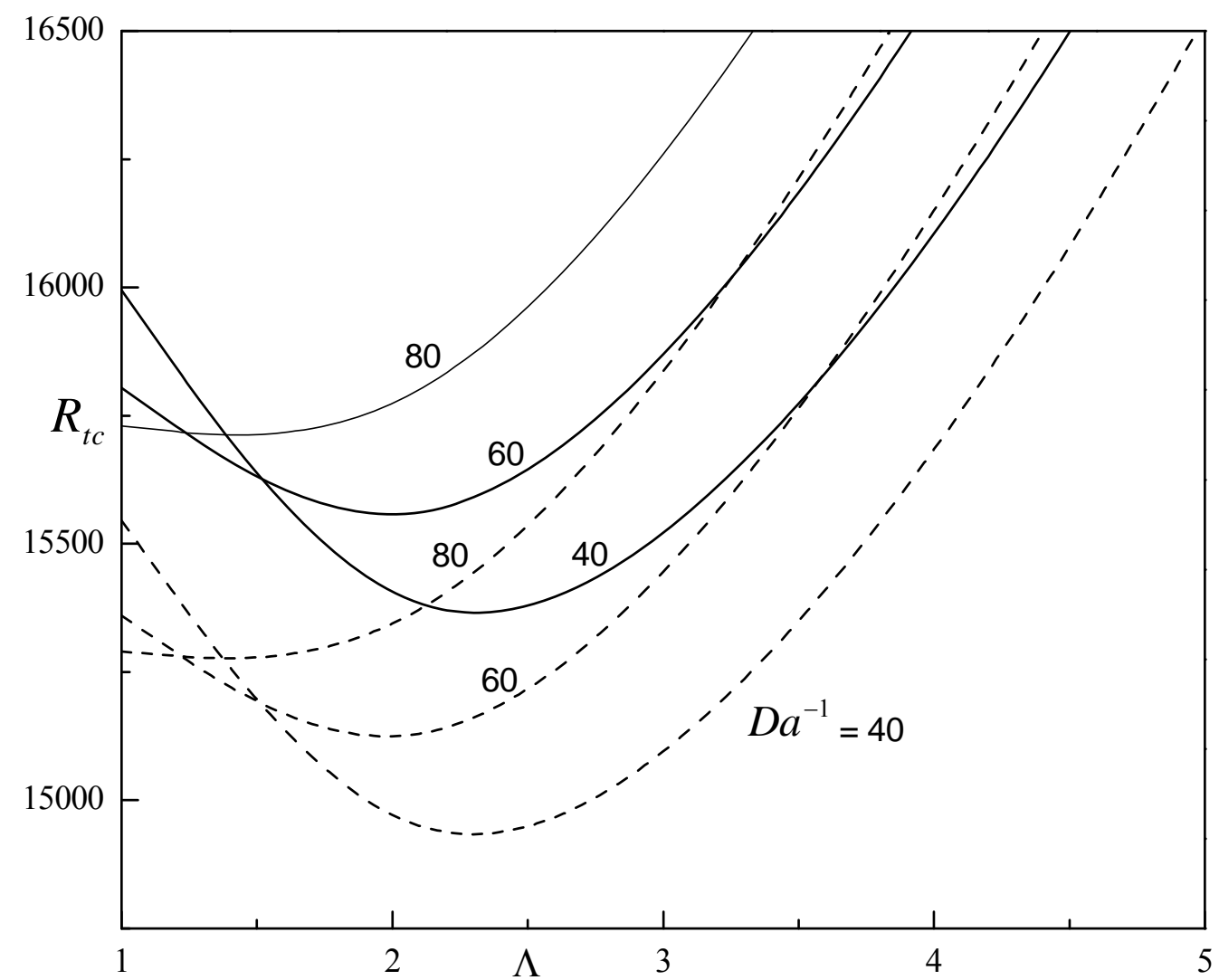

Fig. 4. $R_{t c}$ Vs $\Lambda$ for different $D a^{-1}$ when $T a=10^{5}$ for rigid-rigid boundaries when $R_{e a}=0(-), R_{e a}=500(--)$.

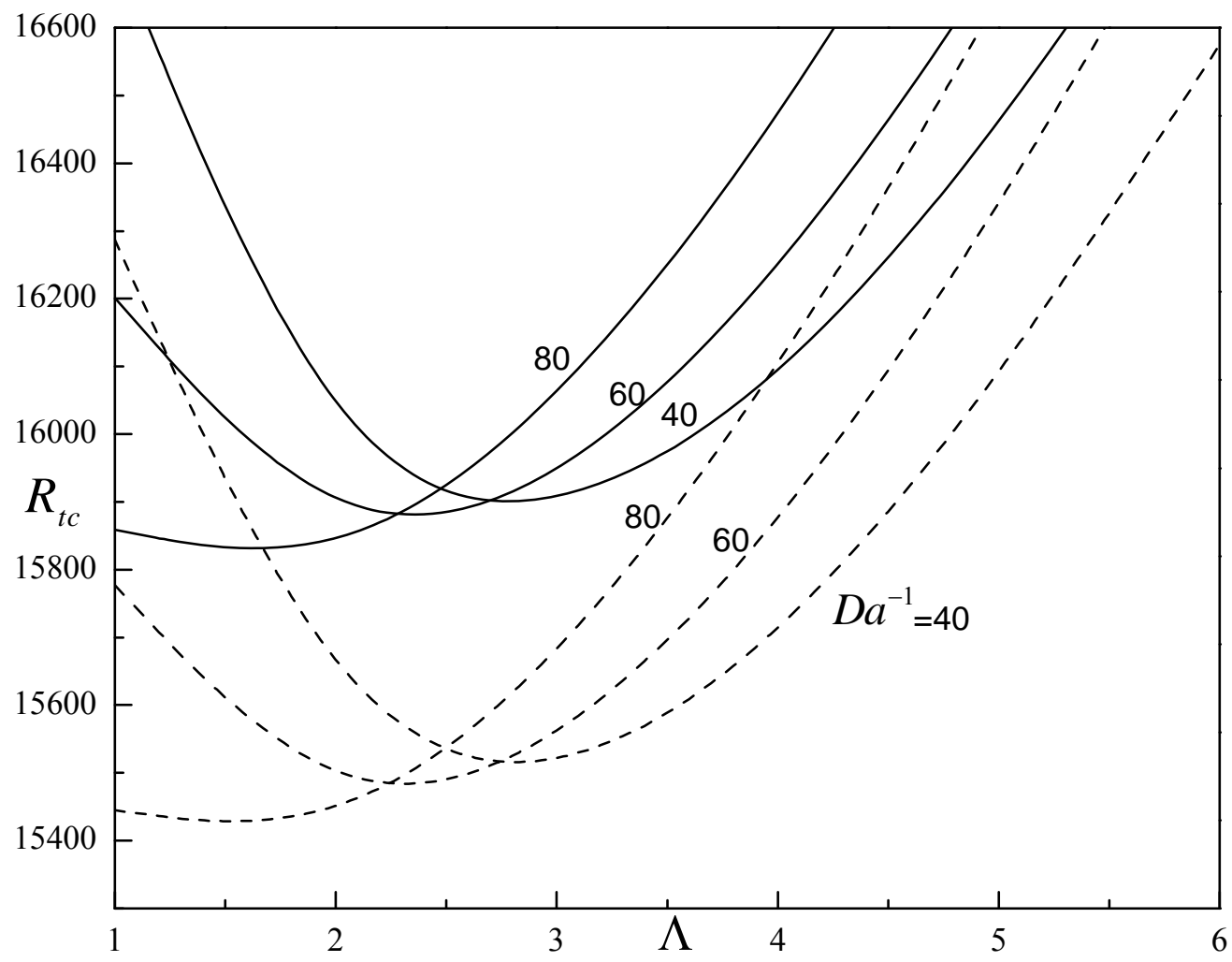

Fig. 5. $R_{t c}$ Vs $\Lambda$ for different $D a^{-1}$ when $T a=10^{5}$ for rigid-free boundaries when $R_{e a}=0(-) ; R_{e a}=500(---)$. 


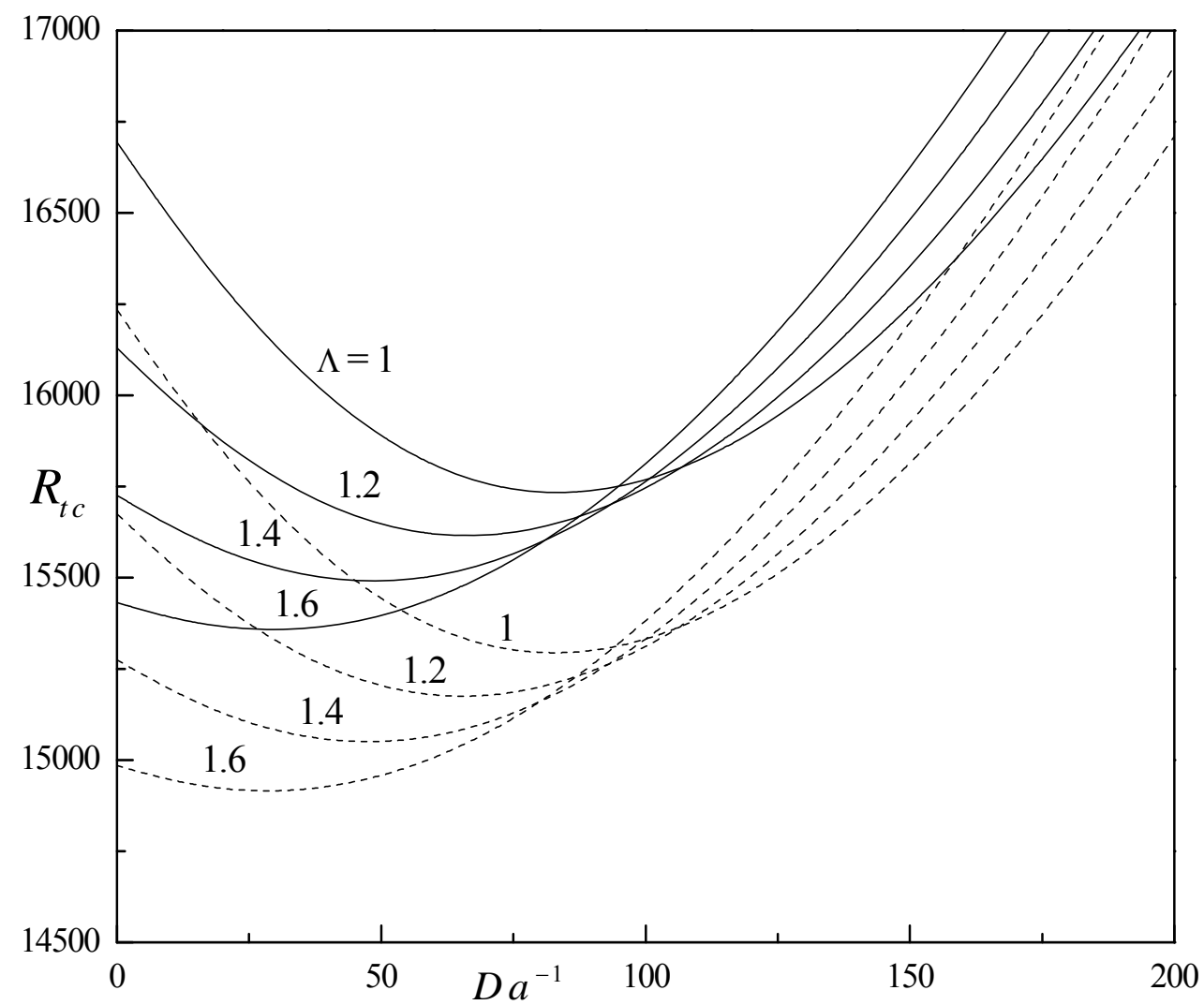

Fig. 6. $R_{t c}$ Vs $D a^{-1}$ for different $\Lambda$ when $T a=10^{5}$ for rigid-rigid boundaries when $R_{e a}=0(-) ; R_{e a}=500(--)$.

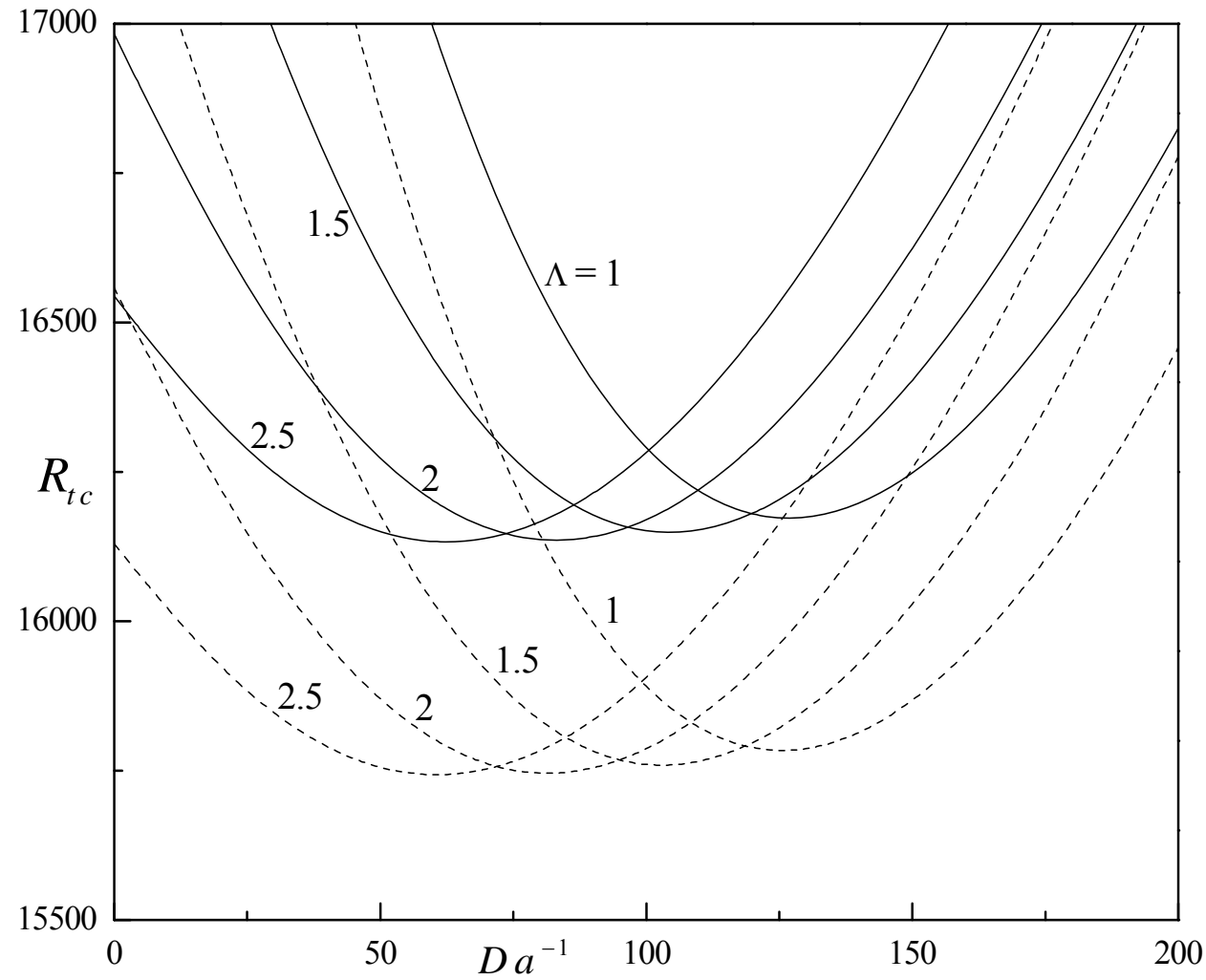

Fig. 7. $R_{t c}$ Vs $D a^{-1}$ for different $\Lambda$ when $T a=10^{5}$ for rigid-free boundaries when $R_{e a}=0(-) ; R_{e a}=500(---)$. 


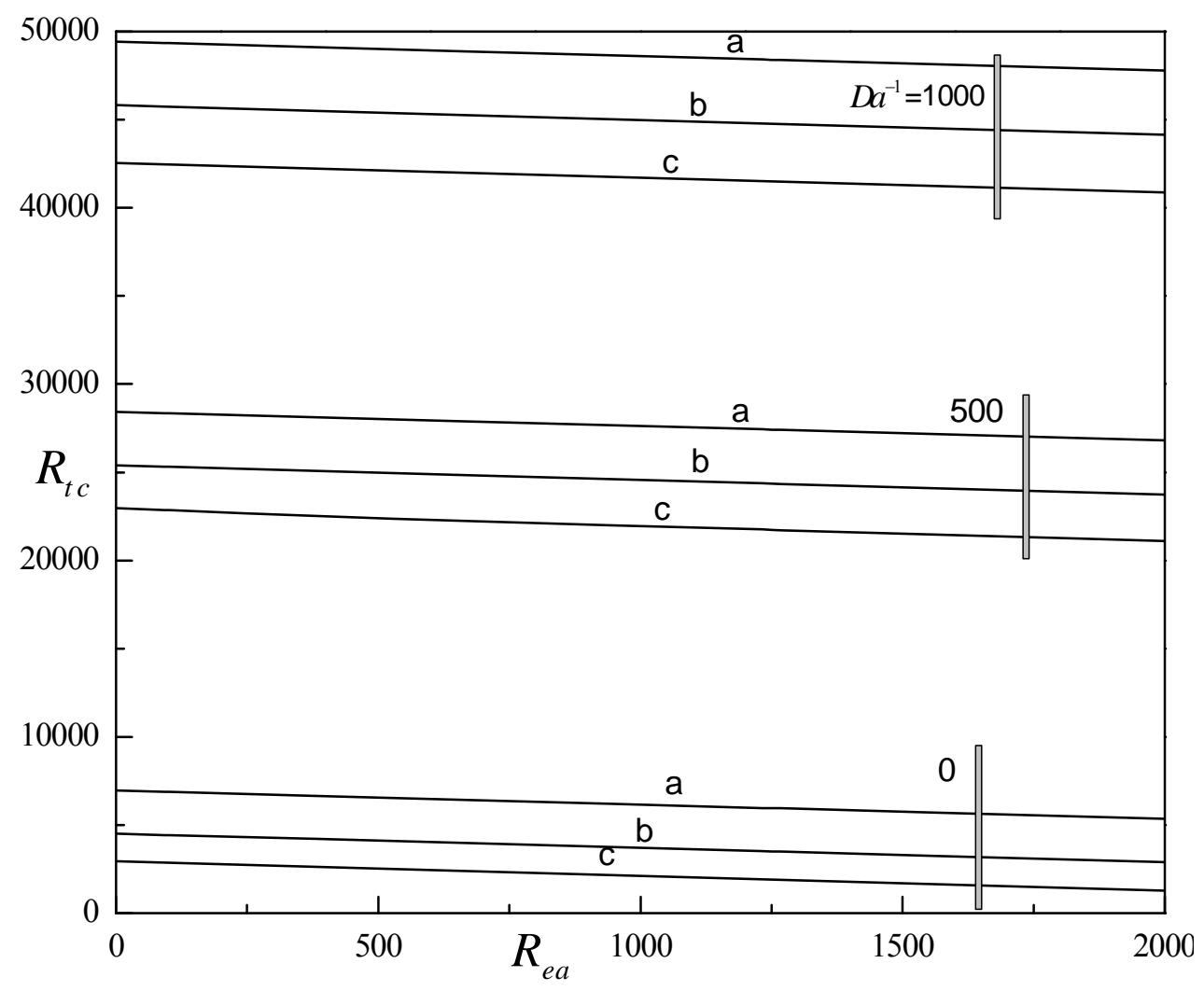

Fig. 8(a). $R_{t c} \operatorname{Vs} R_{e a}$ for different values of $D a^{-1}$ when $\Lambda=4, T a=10^{5}$ for (a) rigid-rigid, (b) rigid-free and (c) free-free boundaries

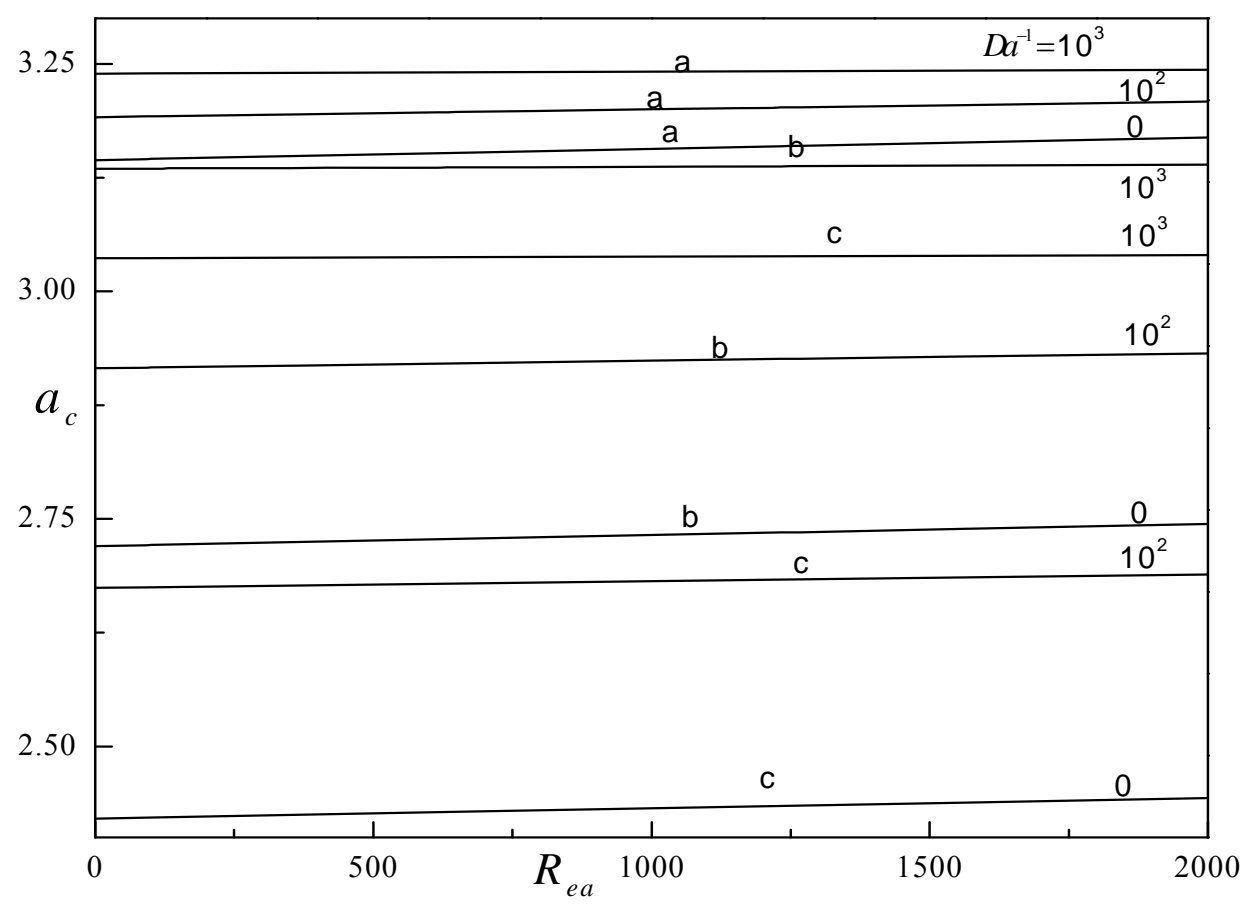

Fig. 8(b). $a_{c} \operatorname{Vs} R_{e a}$ for different values of $D a^{-1}$ when $\Lambda=4, T a=10^{5}$ for (a) rigid-rigid, (b) rigid-free and (c) free-free boundaries 


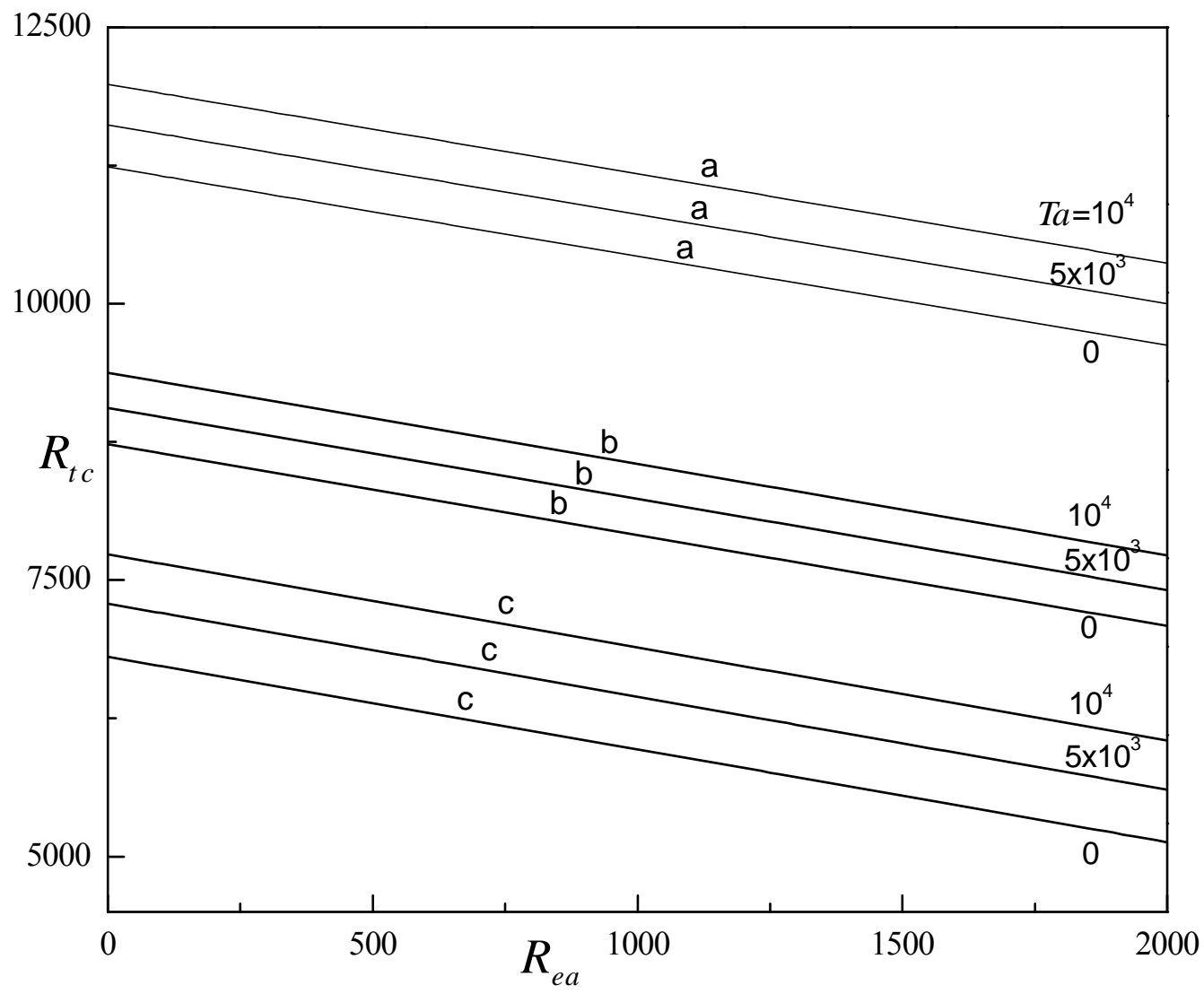

Fig. 9(a). $R_{t c}$ Vs $R_{e a}$ for different values of $T a$ when $\Lambda=4, D a^{-1}=10^{2}$ for (a) rigid-rigid, (b) rigid-free and (c) free-free boundaries

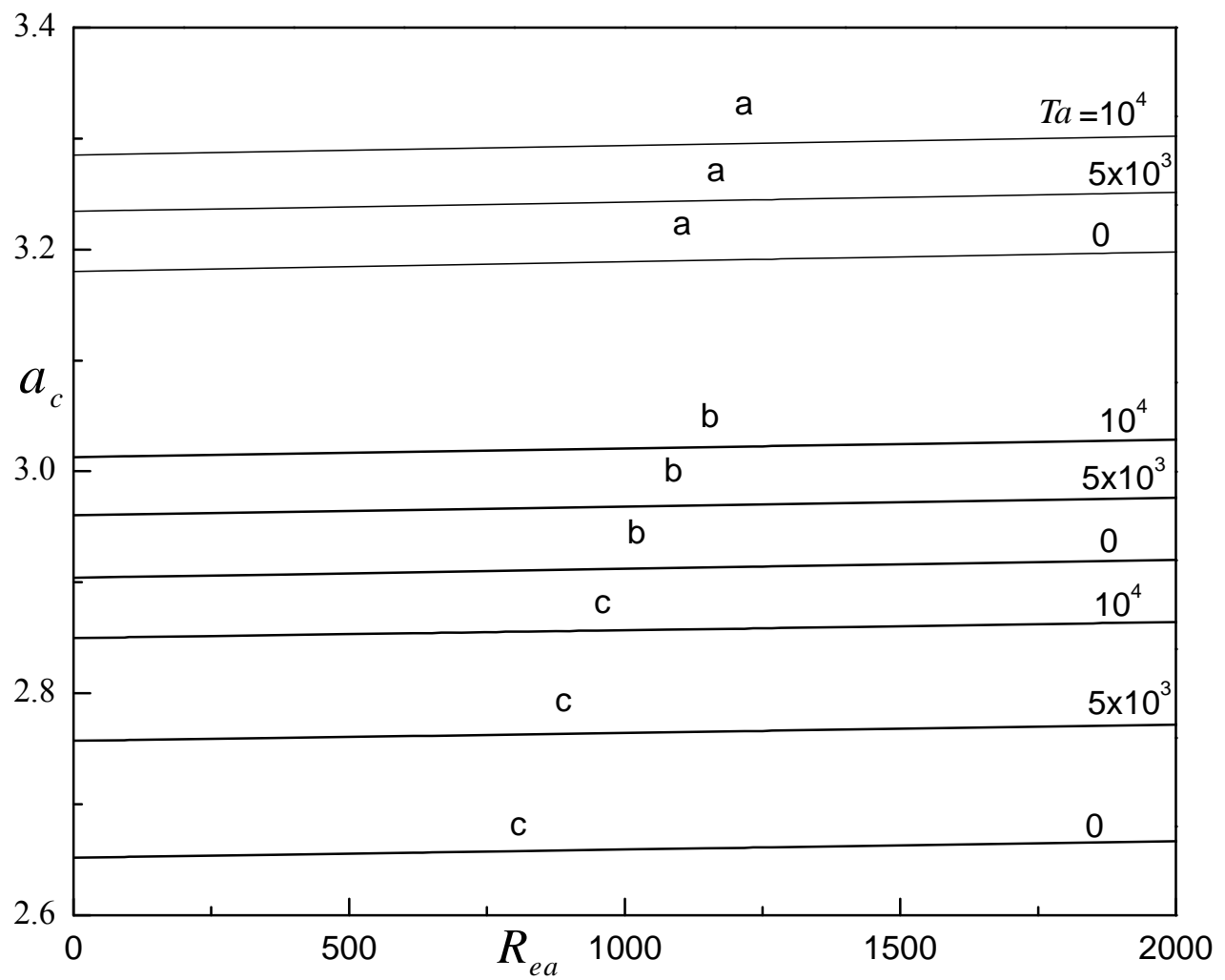

Fig. 9(b). $a_{c} \operatorname{Vs} R_{e a}$ for different values of $T a$ when $\Lambda=4, D a^{-1}=10^{2}$ for (a) rigid-rigid, (b) rigid-free and (c) free-free boundaries 


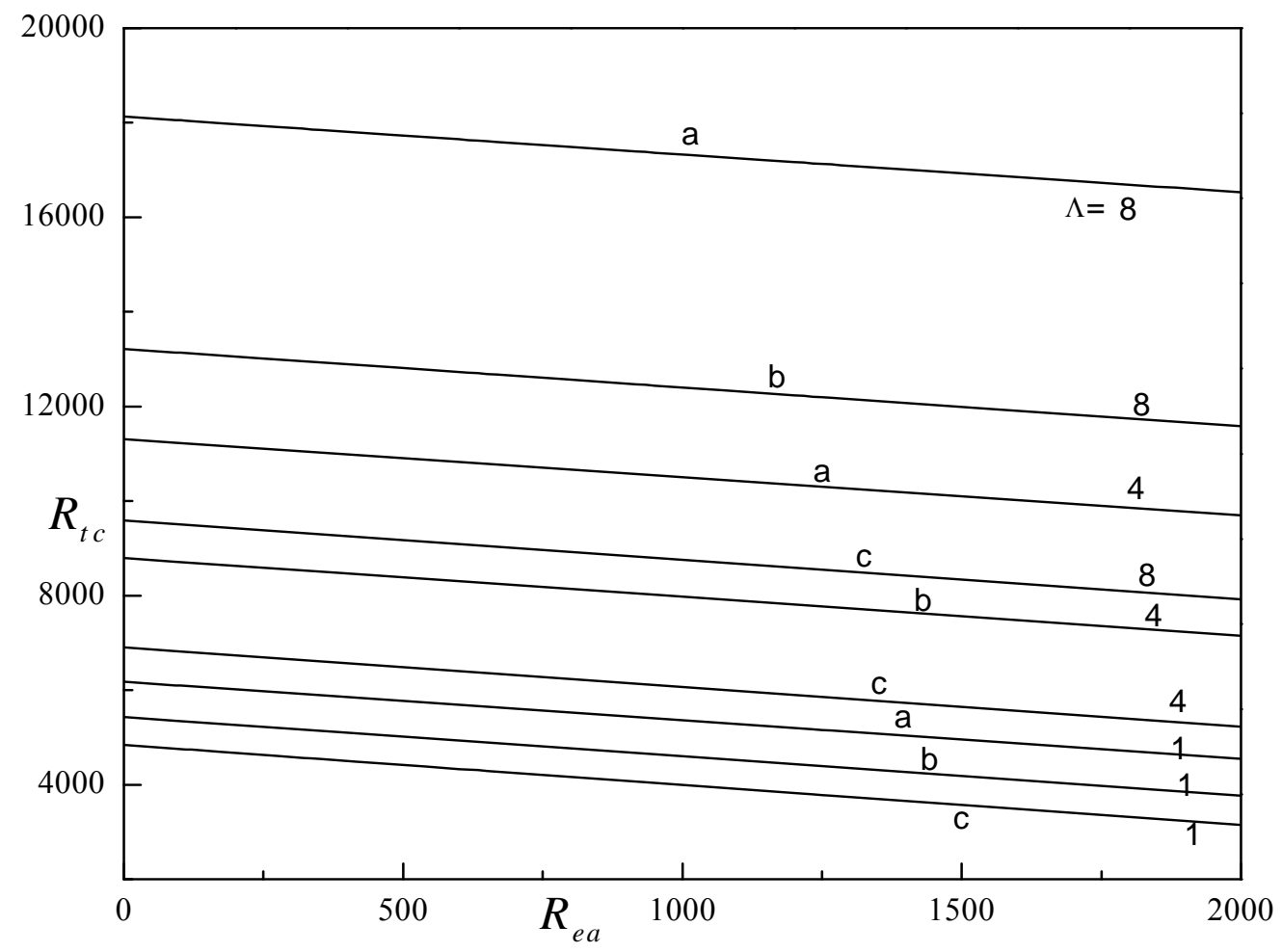

Fig. 10(a). $R_{t c} \operatorname{Vs} R_{e a}$ for different values of $\Lambda$ when $T a=10^{5}, D a^{-1}=10^{2}$ for (a) rigid-rigid, (b) rigid-free and (c) free-free boundaries

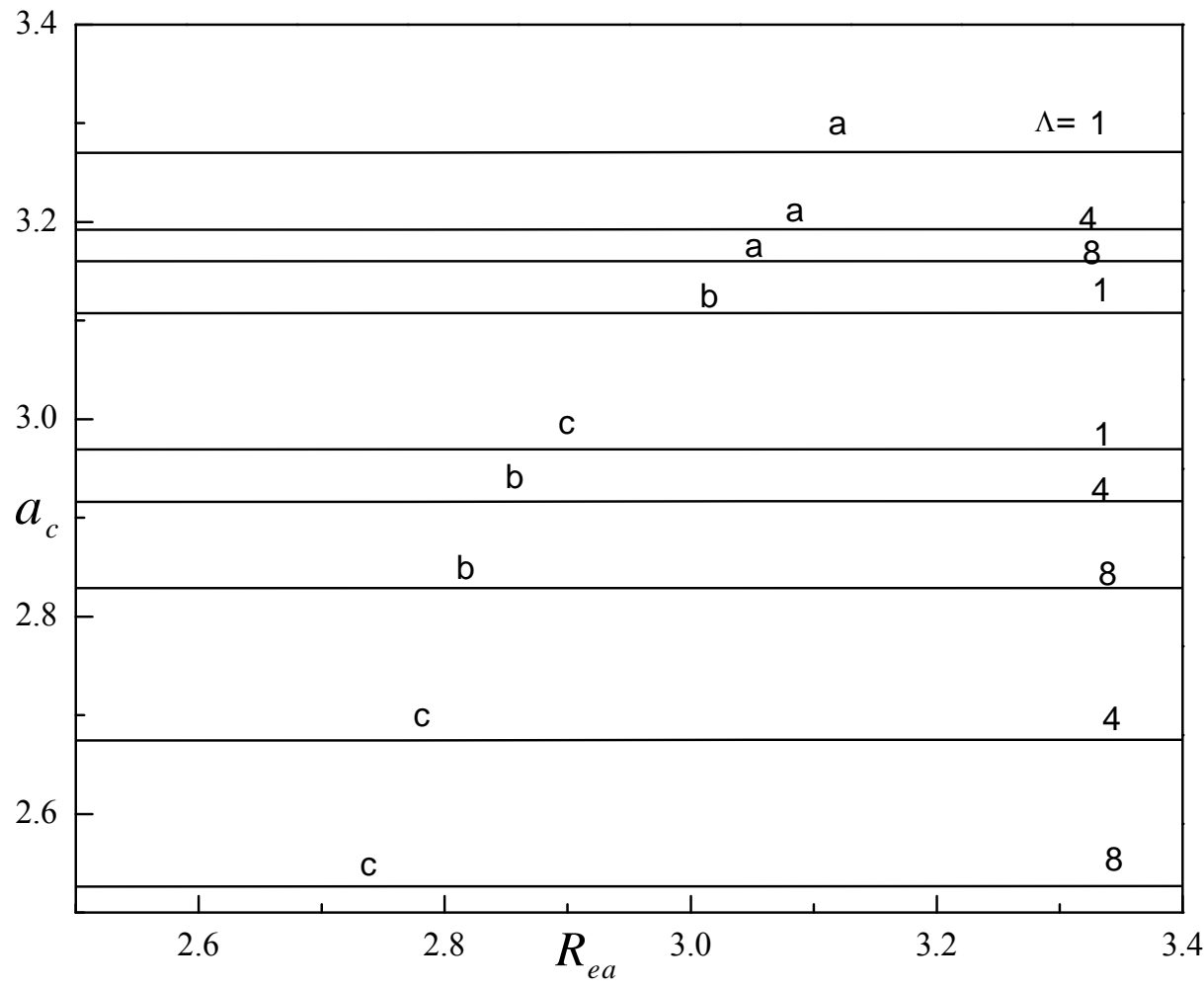

Fig. 10(b). $a_{c}$ Vs $R_{e a}$ for different values of $\Lambda$ when $T a=10^{5}, D a^{-1}=10^{2}$ for (a) rigid-rigid, (b) rigid-free and (c) free-free boundaries 


\begin{tabular}{|c|c|c|c|c|}
\hline \multicolumn{3}{|c|}{ Chandrasekhar [1] } & \multicolumn{2}{c|}{ Present study } \\
\hline$T a$ & $R_{t c}$ & $a_{c}$ & $R_{t c}$ & $a_{c}$ \\
\hline 10 & 1713.0 & 3.10 & 1712.67 & 3.12087 \\
\hline 100 & 1756.6 & 3.15 & 1756.35 & 3.16 \\
\hline 500 & 1940.5 & 3.30 & 1940.2 & 3.31926 \\
\hline 1000 & 2151.7 & 3.50 & 2151.34 & 3.48 \\
\hline 2000 & 2530.5 & 3.75 & 2530.13 & 3.74693 \\
\hline 5000 & 3469.2 & 4.25 & 3468.52 & 4.26 \\
\hline 10000 & 4713.1 & 4.80 & 4712.06 & 4.7849 \\
\hline
\end{tabular}

Table 1. Values of $R_{t c}$ and $a_{c}$ for different values of $T a$ for rigid-rigid boundaries

\begin{tabular}{|c|c|c|c|c|}
\hline \multicolumn{3}{|c|}{ Roberts [27] } & \multicolumn{2}{c|}{ Present Study } \\
\hline$R_{t}$ & $R_{\text {eac }}$ & $a_{c}$ & $R_{\text {eac }}^{s}$ & $a_{c}$ \\
\hline-1000 & 3370.077 & 3.2945 & 3370.08 & 3.29446 \\
\hline-500 & 2749.868 & 3.2598 & 2749.87 & 3.25983 \\
\hline 0 & 2128.696 & 3.2260 & 2128.696 & 3.22596 \\
\hline 500 & 1506.573 & 3.1929 & 1506.57 & 3.19287 \\
\hline 1000 & 883.517 & 3.1606 & 883.517 & 1506.57 \\
\hline 1707.762 & 0 & 3.1162 & 0 & 3.11621 \\
\hline
\end{tabular}

Table 2. Values of $R_{\text {eac }}$ and $a_{c}$ for different values of $R_{t}$ for rigid-rigid boundaries 


\section{Free-free boundaries}

\begin{tabular}{|c|c|c|c|c|c|c|}
\hline \multirow{2}{*}{$\Lambda$} & \multicolumn{2}{|c|}{$R_{e a}=0$} & \multicolumn{2}{c|}{$R_{e a}=500$} & \multicolumn{2}{c|}{$R_{e a}=1000$} \\
\cline { 2 - 7 } & $\left(R_{t c}\right)_{\min }$ & $D a_{\min }^{-1}$ & $\left(R_{t c}\right)_{\min }$ & $D a_{\min }^{-1}$ & $\left(R_{t c}\right)_{\min }$ & $D a_{\min }^{-1}$ \\
\hline 1 & 16217.4 & 152.96 & 15882.9 & 151.31 & 15546.2 & 149.66 \\
\hline 2 & 16217.4 & 123.35 & 15882.9 & 121.29 & 15546.2 & 119.23 \\
\hline 3 & 16217.4 & 93 & 15882.9 & 91.17 & 15546.2 & 88.70 \\
\hline 4 & 16217.4 & 64 & 15882.9 & 61.26 & 15546.2 & 58.37 \\
\hline 5 & 16217.4 & 34.53 & 15882.9 & 31.24 & 15546.2 & 27.94 \\
\hline
\end{tabular}

\section{$\underline{\text { Rigid-rigid boundaries }}$}

\begin{tabular}{|c|c|c|c|c|c|c|}
\hline \multirow{2}{*}{$\Lambda$} & \multicolumn{2}{|c|}{$R_{e a}=0$} & \multicolumn{2}{c|}{$R_{e a}=500$} & \multicolumn{2}{c|}{$R_{e a}=1000$} \\
\cline { 2 - 7 } & $\left(R_{t c}\right)_{\min }$ & $D a_{\min }^{-1}$ & $\left(R_{t c}\right)_{\min }$ & $D a_{\min }^{-1}$ & $\left(R_{t c}\right)_{\min }$ & $D a_{\min }^{-1}$ \\
\hline 1 & 15728 & 83.34 & 15289.4 & 82.65 & 14849.8 & 81.95 \\
\hline 1.2 & 15611 & 66.24 & 15171 & 65.43 & 14731.4 & 64.63 \\
\hline 1.4 & 15487 & 48.44 & 15047 & 47.54 & 14606 & 46.62 \\
\hline 1.6 & 15354 & 29.72 & 14913 & 28.65 & 14472 & 27.58 \\
\hline 1.8 & 15211 & 9.62 & 14768 & 8.36 & 14325.3 & 7.078 \\
\hline
\end{tabular}

\section{$\underline{\text { Rigid-free boundaries }}$}

\begin{tabular}{|c|c|c|c|c|c|c|}
\hline \multirow{2}{*}{$\Lambda$} & \multicolumn{2}{|c|}{$R_{e a}=0$} & \multicolumn{2}{c|}{$R_{e a}=500$} & \multicolumn{2}{c|}{$R_{e a}=1000$} \\
\cline { 2 - 7 } & $\left(R_{t c}\right)_{\min }$ & $D a_{\min }^{-1}$ & $\left(R_{t c}\right)_{\min }$ & $D a_{\min }^{-1}$ & $\left(R_{t c}\right)_{\min }$ & $D a_{\min }^{-1}$ \\
\hline 1 & 16152 & 126.07 & 15763.5 & 124.84 & 15373 & 123.61 \\
\hline 2 & 16119 & 82.71 & 15729.5 & 81.14 & 15338 & 79.57 \\
\hline 3 & 16127 & 42.64 & 15737.4 & 40.78 & 15346 & 38.92 \\
\hline 4 & 16174 & 5.441 & 15785.7 & 3.328 & 15395 & 1.213 \\
\hline
\end{tabular}

Table 3. Values of $\left(R_{t c}\right)_{\min }$ and $D a_{\min }^{-1}$ for different values of $\Lambda$ and $R_{e}$ when $T a=10^{5}$ 


\section{Free-Free boundaries}

\begin{tabular}{|c|c|c|c|c|c|c|}
\hline \multirow{2}{*}{$D a^{-1}$} & \multicolumn{2}{|c|}{$R_{e a}=0$} & \multicolumn{2}{c|}{$R_{e a}=500$} & \multicolumn{2}{c|}{$R_{e a}=1000$} \\
\cline { 2 - 7 } & $\left(R_{t c}\right)_{\min }$ & $\Lambda_{\min }$ & $\left(R_{t c}\right)_{\min }$ & $\Lambda_{\min }$ & $\left(R_{t c}\right)_{\min }$ & $\Lambda_{\min }$ \\
\hline 10 & 16217.4 & 5.828 & 15882.9 & 5.708 & 15546 & 5.589 \\
\hline 20 & 16217.4 & 5.491 & 15882.9 & 5.375 & 15546 & 5.261 \\
\hline 30 & 16217.4 & 5.153 & 15882.9 & 5.041 & 15546 & 4.932 \\
\hline 50 & 16217.4 & 4.477 & 15882.9 & 4.375 & 15546 & 4.275 \\
\hline 100 & 16217.4 & 2.789 & 15882.9 & 2.710 & 15546 & 2.632 \\
\hline 150 & 16217.4 & 1.100 & 15882.9 & 1.044 & 15546 & 0.989 \\
\hline
\end{tabular}

\section{$\underline{\text { Rigid-rigid boundaries }}$}

\begin{tabular}{|c|c|c|c|c|c|c|}
\hline \multirow{2}{*}{$D a^{-1}$} & \multicolumn{2}{|c|}{$R_{e a}=0$} & \multicolumn{2}{c|}{$R_{e a}=500$} & \multicolumn{2}{c|}{$R_{e a}=1000$} \\
\cline { 2 - 7 } & $\left(R_{t c}\right)_{\min }$ & $\Lambda_{\min }$ & $\left(R_{t c}\right)_{\min }$ & $\Lambda_{\min }$ & $\left(R_{t c}\right)_{\min }$ & $\Lambda_{\min }$ \\
\hline 10 & 14953 & 2.596 & 14520 & 2.581 & 14087 & 2.565 \\
\hline 20 & 15052 & 2.444 & 14618 & 2.428 & 14185 & 2.413 \\
\hline 30 & 15147 & 2.289 & 14714 & 2.274 & 14281 & 2.259 \\
\hline 50 & 15331 & 1.99 & 14898 & 1.964 & 14464 & 1.951 \\
\hline 100 & 15743 & 1.196 & 15308 & 1.186 & 14874 & 1.176 \\
\hline 150 & 16118 & 0.578 & 15684 & 0.574 & 15250 & 0.569 \\
\hline
\end{tabular}

\section{$\underline{\text { Rigid-free boundaries }}$}

\begin{tabular}{|c|c|c|c|c|c|c|}
\hline \multirow{2}{*}{$D a^{-1}$} & \multicolumn{2}{|c|}{$R_{e a}=0$} & \multicolumn{2}{c|}{$R_{e a}=500$} & \multicolumn{2}{c|}{$R_{e a}=1000$} \\
\cline { 2 - 7 } & $\left(R_{t c}\right)_{\min }$ & $\Lambda_{\min }$ & $\left(R_{t c}\right)_{\min }$ & $\Lambda_{\min }$ & $\left(R_{t c}\right)_{\min }$ & $\Lambda_{\min }$ \\
\hline 10 & 16159.2 & 3.651 & 15767 & 3.603 & 15374 & 3.556 \\
\hline 20 & 16146.2 & 3.428 & 15754 & 3.382 & 15361 & 3.336 \\
\hline 30 & 16135.7 & 3.204 & 15744 & 3.159 & 15352 & 3.116 \\
\hline 50 & 16222 & 2.276 & 15731 & 2.716 & 15340 & 2.676 \\
\hline 100 & 16126 & 1.646 & 15738 & 1.616 & 15348 & 1.588 \\
\hline
\end{tabular}

Table 4. Values of $\left(R_{t c}\right)_{\min }$ and $\Lambda_{\min }$ for different values of $D a^{-1}$ and $R_{e a}$ when $T a=10^{5}$ 


\section{Free-Free boundaries}

\begin{tabular}{|c|c|c|c|c|c|c|}
\hline \multirow{2}{*}{$\Lambda$} & \multicolumn{2}{|c|}{$R_{e a}=0$} & \multicolumn{2}{c|}{$R_{e a}=500$} & \multicolumn{2}{c|}{$R_{e a}=1000$} \\
\cline { 2 - 7 } & $\left(R_{t c}\right)_{\min }$ & $D a_{\min }^{-1}$ & $\left(R_{t c}\right)_{\min }$ & $D a_{\min }^{-1}$ & $\left(R_{t c}\right)_{\min }$ & $D a_{\min }^{-1}$ \\
\hline 1 & 22934.9 & 228.96 & 22600.7 & 227.056 & 22265 & 225.52 \\
\hline 2 & 22934.9 & 198.99 & 22600.7 & 197.16 & 22265 & 195.33 \\
\hline 3 & 22934.9 & 169.37 & 22600.7 & 167.27 & 22265 & 165.15 \\
\hline 4 & 22934.9 & 139.76 & 22600.7 & 137.36 & 22265 & 104.77 \\
\hline 5 & 22934.9 & 110.16 & 22600.7 & 107.47 & 22265 & 74.59 \\
\hline 6 & 22934.9 & 80.54 & 22600.7 & 77.57 & 22265 & 44.40 \\
\hline
\end{tabular}

\section{$\underline{\text { Rigid-rigid boundaries }}$}

\begin{tabular}{|c|c|c|c|c|c|c|}
\hline \multirow{2}{*}{$\Lambda$} & \multicolumn{2}{|c|}{$R_{e a}=0$} & \multicolumn{2}{c|}{$R_{e a}=500$} & \multicolumn{2}{c|}{$R_{e a}=1000$} \\
\cline { 2 - 7 } & $\left(R_{t c}\right)_{\min }$ & $D a_{\min }^{-1}$ & $\left(R_{t c}\right)_{\min }$ & $D a_{\min }^{-1}$ & $\left(R_{t c}\right)_{\min }$ & $D a_{\min }^{-1}$ \\
\hline 1 & 22484 & 152.84 & 22046.8 & 152.244 & 21607.7 & 151.66 \\
\hline 1.2 & 22366.8 & 135.94 & 21927.8 & 135.302 & 21488.8 & 134.66 \\
\hline 1.4 & 22252.1 & 119.09 & 21812.8 & 118.40 & 21373.3 & 117.703 \\
\hline 1.6 & 22136 & 102.05 & 21696.2 & 101.292 & 21253.3 & 100.53 \\
\hline 1.8 & 22015 & 84.63 & 21575.5 & 83.79 & 21351 & 82.96 \\
\hline 2 & 21890 & 66.68 & 21449.2 & 65.76 & 21008.3 & 64.83 \\
\hline
\end{tabular}

\section{$\underline{\text { Rigid-free boundaries }}$}

\begin{tabular}{|c|c|c|c|c|c|c|}
\hline \multirow{2}{*}{$\Lambda$} & \multicolumn{2}{|c|}{$R_{e a}=0$} & \multicolumn{2}{c|}{$R_{e a}=500$} & \multicolumn{2}{c|}{$R_{e a}=1000$} \\
\cline { 2 - 7 } & $\left(R_{t c}\right)_{\min }$ & $D a_{\min }^{-1}$ & $\left(R_{t c}\right)_{\min }$ & $D a_{\min }^{-1}$ & $\left(R_{t c}\right)_{\min }$ & $D a_{\min }^{-1}$ \\
\hline 1 & 22865 & 197.52 & 22477 & 196.32 & 22088 & 195.18 \\
\hline 2 & 22816 & 152.21 & 22427 & 150.84 & 22037 & 149.46 \\
\hline 3 & 22714 & 109.87 & 22404 & 108.27 & 22014 & 106.66 \\
\hline 4 & 22801 & 69.73 & 22411 & 67.92 & 22021 & 66.11 \\
\hline 5 & 22836 & 31.62 & 22466 & 29.63 & 22056 & 27.63 \\
\hline
\end{tabular}

Table 5. Values of $\left(R_{t c}\right)_{\min }$ and $D a_{\min }^{-1}$ for different values of $\Lambda$ and $R_{e a}$ when $T a=2 \times 10^{5}$ 


\section{Free-Free boundaries}

\begin{tabular}{|c|c|c|c|c|c|c|}
\hline \multirow{2}{*}{$D a^{-1}$} & \multicolumn{2}{|c|}{$R_{e a}=0$} & \multicolumn{2}{c|}{$R_{e a}=500$} & \multicolumn{2}{c|}{$R_{e a}=1000$} \\
\cline { 2 - 7 } & $\left(R_{t c}\right)_{\min }$ & $\Lambda_{\min }$ & $\left(R_{t c}\right)_{\min }$ & $\Lambda_{\min }$ & $\left(R_{t c}\right)_{\min }$ & $\Lambda_{\min }$ \\
\hline 10 & 22934.9 & 8.382 & 22600 & 8.260 & 22265 & 8.140 \\
\hline 20 & 22934.9 & 8.045 & 22600 & 7.925 & 22265 & 7.808 \\
\hline 30 & 22934.9 & 7.706 & 22600 & 7.591 & 22265 & 7.477 \\
\hline 50 & 22934.9 & 7.032 & 22600 & 6.922 & 22265 & 6.814 \\
\hline 100 & 22934.9 & 5.342 & 22600 & 5.249 & 22265 & 5.158 \\
\hline 150 & 22934.9 & 3.654 & 22600 & 3.578 & 22265 & 3.502 \\
\hline
\end{tabular}

\section{$\underline{\text { Rigid-rigid boundaries }}$}

\begin{tabular}{|c|c|c|c|c|c|c|}
\hline \multirow{2}{*}{$D a^{-1}$} & \multicolumn{2}{|c|}{$R_{e a}=0$} & \multicolumn{2}{c|}{$R_{e a}=500$} & \multicolumn{2}{c|}{$R_{e a}=1000$} \\
\cline { 2 - 7 } & $\left(R_{t c}\right)_{\min }$ & $\Lambda_{\min }$ & $\left(R_{t c}\right)_{\min }$ & $\Lambda_{\min }$ & $\left(R_{t c}\right)_{\min }$ & $\Lambda_{\min }$ \\
\hline 10 & 21106 & 3.735 & 20673 & 3.719 & 20240 & 3.703 \\
\hline 20 & 21205 & 3.583 & 20772 & 3.567 & 20339 & 3.551 \\
\hline 30 & 21303 & 3.429 & 20870 & 3.414 & 20436 & 3.398 \\
\hline 50 & 21493 & 3.120 & 21060 & 3.106 & 20626 & 3.091 \\
\hline 100 & 21935 & 2.334 & 21501 & 2.325 & 21067 & 2.312 \\
\hline 150 & 22327 & 1.564 & 21893 & 1.554 & 21459 & 1.544 \\
\hline
\end{tabular}

\section{$\underline{\text { Rigid-free boundaries }}$}

\begin{tabular}{|c|c|c|c|c|c|c|}
\hline \multirow{2}{*}{$D a^{-1}$} & \multicolumn{2}{|c|}{$R_{e a}=0$} & \multicolumn{2}{c|}{$R_{e a}=500$} & \multicolumn{2}{c|}{$R_{e a}=1000$} \\
\cline { 2 - 7 } & $\left(R_{t c}\right)_{\min }$ & $\Lambda_{\min }$ & $\left(R_{t c}\right)_{\min }$ & $\Lambda_{\min }$ & $\left(R_{t c}\right)_{\min }$ & $\Lambda_{\min }$ \\
\hline 10 & 22858 & 5.254 & 22466 & 5.206 & 22073 & 5.158 \\
\hline 20 & 22844 & 5.034 & 22452 & 4.987 & 22060 & 4.940 \\
\hline 30 & 22832 & 4.808 & 22440 & 4.762 & 22048 & 4.717 \\
\hline 50 & 22812 & 4.359 & 22421 & 4.316 & 22030 & 4.273 \\
\hline 100 & 22793 & 3.242 & 22404 & 3.206 & 22013 & 3.017 \\
\hline 150 & 22812 & 2.142 & 22424 & 2.112 & 22035 & 2.083 \\
\hline
\end{tabular}

Table 6.Values of $\left(R_{t c}\right)_{\min }$ and $\Lambda_{\min }$ for different values of $D a^{-1}$ and $R_{e a}$ when $T a=2 \times 10^{5}$ 